\title{
MALTA UNIVERSITY.
}

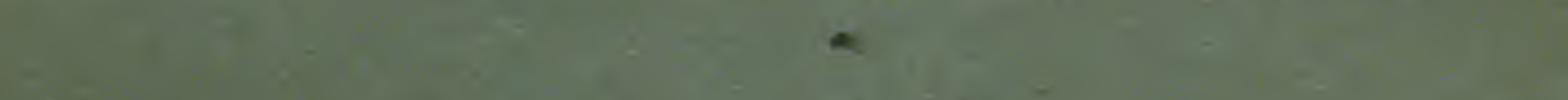

Arcotti Botanic Gardens

GENERAL CATALOGUE.

1924-25.

a 8 \%

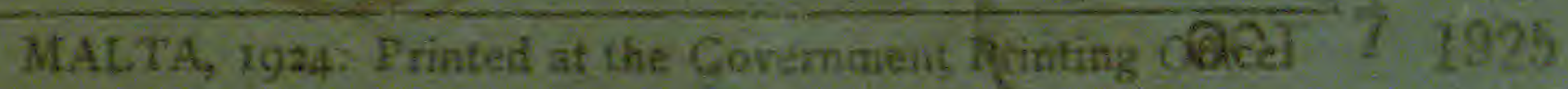

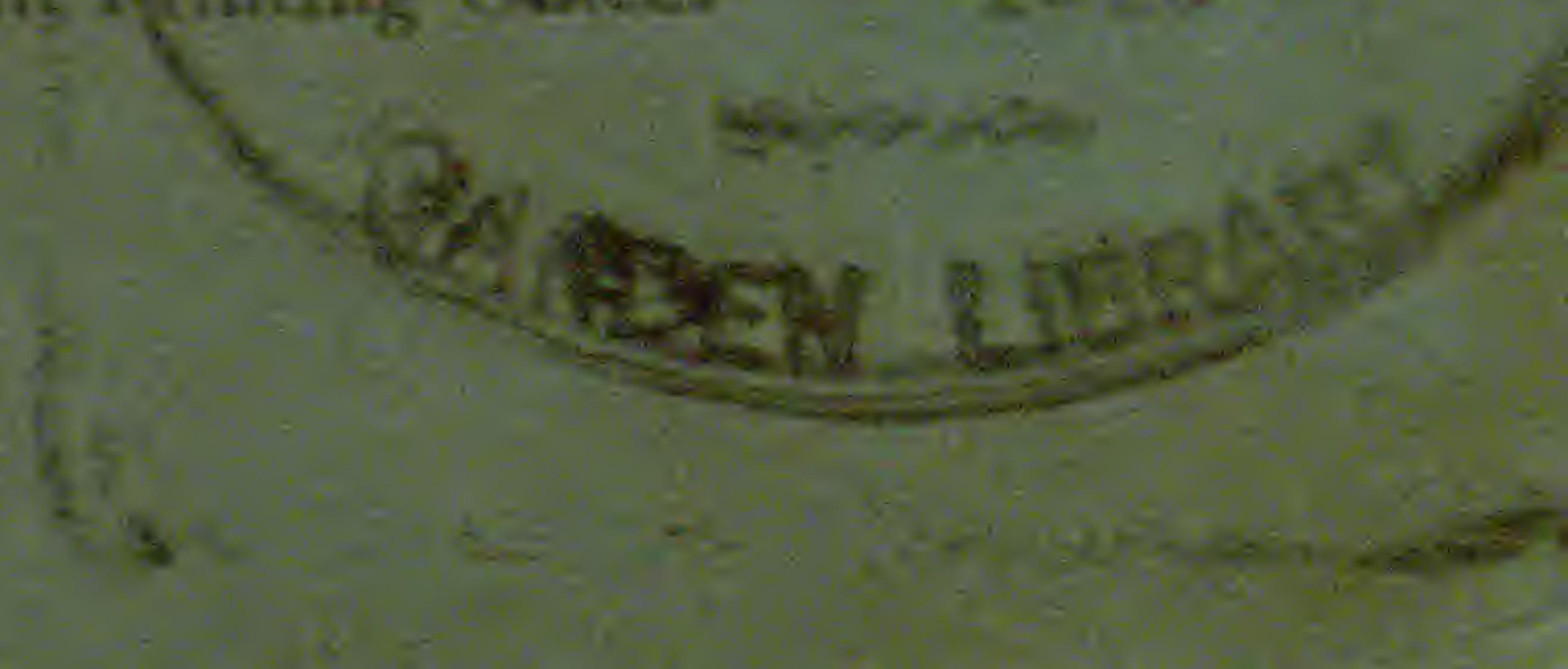


$A \in \beta 45$

\section{MALTA UNIVERSITY.}

\section{Arcotti Botanic Gardens}

GENERAL CATALOGUE.

$$
\text { 1924-25. }
$$

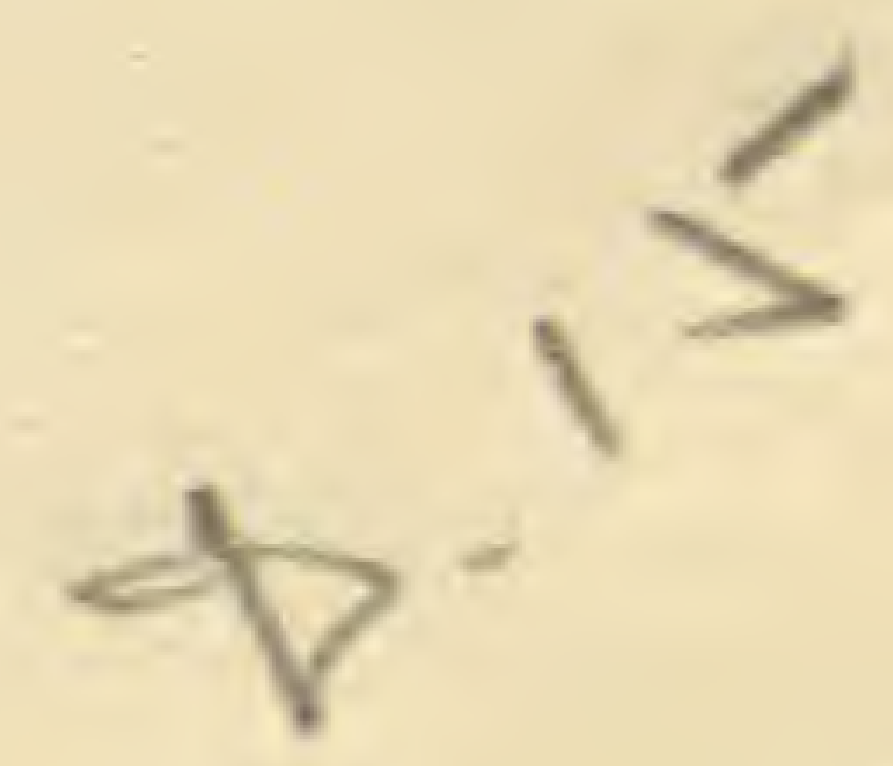

MALTA, 1924: Printed at the Government Printing Officc. 
$\mathrm{N}=$ Indigenous in the Maltese Islands

$t=$ Seeds available for exchange 


\title{
DICOTYLEDONES.
}

Acanthaceae.

$\mathrm{N}+$ Acanthus mollis, Linn.

$\mathrm{N}$

\author{
var, alliflorus, Bors.
}

Schottii, Linn.

Adhatoda Vasica, Nees.

$\dagger$ Anisacanthus Wrigh:ii, A. Gray.

Aphelandra aurantiaca, Lindl.

Fittonia Verschaffelti, E. Cotm.

" argyroneurd, E. Coem.

Justicia coccinea, Aubl.

" flava, Kurtz.

$\dagger \quad$ ventricosa, Wail.

Peristrophe speciosa, Nees.

Sanchezia nobilis, Hook.

Ruellia Blumei, Steud.

\section{Amaranthaceae.}

Alternanthera Achyrantha, R. Br.

sessilis, R. Br.

$\dagger$ Amaranthus blitum, Lins.

caudatus, Linn.

gangeticus, Linn.

paniculatus, linn.

retroflexus, Limn.

Salicifolius, Hort. Veitch.

hypochondriarhus, Linn.

polygonoides, finn.

$\mathrm{N} \dagger$ quadricolor, Hort.

Bosea Yerva-mora, Linn.

† Celosia cristata, Linn.

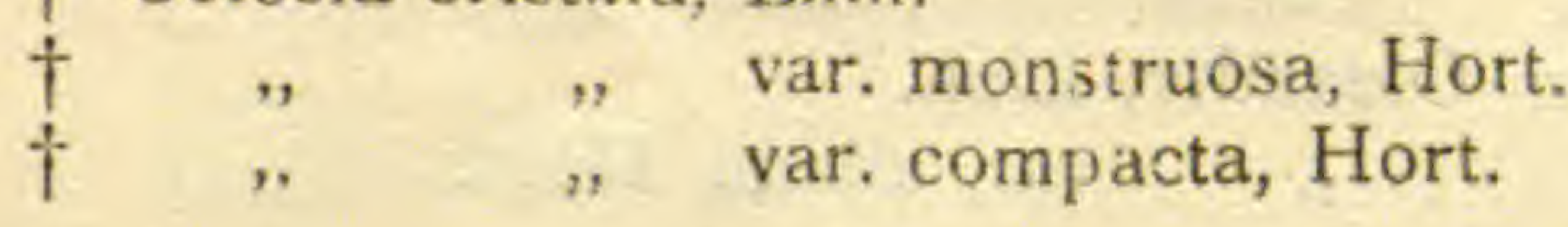




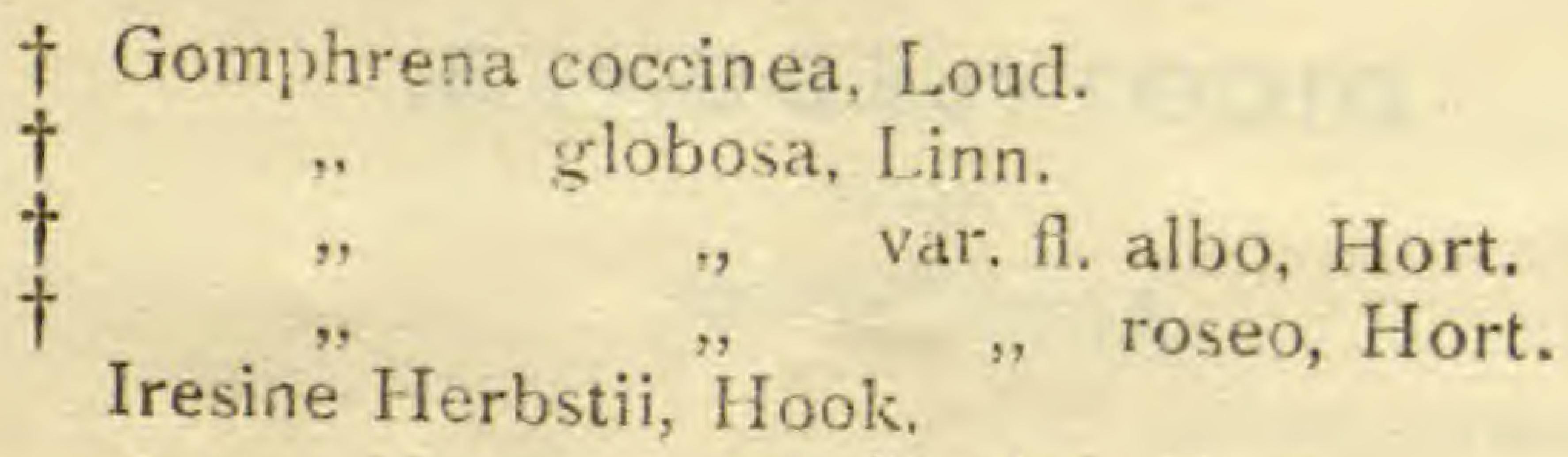

" var, bicolor, Hort.

$\dagger$ Ochnida cannabina, Linn.

\section{Ampelidaceae.}

Ampelopsis Veitchli, H» rt.

Vitis Berlandieri, Planch.

$\dagger$ " vinifera, Linn.

\section{Anacardiaceae.}

N Pistacia Lentiscus, Linn,

Corynecarpus laevigata, Fiorst.

vera, Linn.

$\dagger$ Pleiogynum Solandri, Engl.

Quebrachia Lorentzii, Grisb.

N Rhus coriaria, Linn.

" toxicodendron, Linn.

". vernicifera, D.C.

$\dagger$ Schinus molle, Linn.

$\dagger \quad$ " terebinthifolius, Rac'di.

\section{Anonaceae.}

$\dagger$ Anona cherimolia, Mill.

\section{Apocynaceae.}

Amsonia Tabernæmontana, Walt. Apocynum androsæmifolium, Linn.

† Nerium Oleander, Linn. 
$\dagger$ Nerium Oleander, var, album plenum, Hort

$\dagger \quad " \quad$ " var. rubrum, Hort.

Strophanthus Righierii, Linn.

var. fol. varieg., Hort.

$\dagger$ Thevetia nereifolia, Juss. ex Steud.

Vinca major, Linn.

† " " $\quad$ rosea, Linn.
$\dagger \quad, \quad$ varieg., Hort.
$\dagger$ alba, Hort.

\section{Araliaceae.}

Acanthopanax sessiliflorum, Seem.

Aralia elegantissima, Veitch.

" Sieboldi, Hort.

" Veitchii, Hort. ex. T. Moore.

Spec.

$\mathrm{N} \dagger$ Hedera Helix, Linn.

" arborea, Sw. fl. Ind.

Meryta Denhamii, Seem.

$\dagger$ Sciadophylum digitatum, G. Don.

\section{Aristolochiaceae.}

$\dagger$ Aristolochia fimbriata, Cham.

$\dagger \quad$ " elegans, Mast.

". grandiflora, Arruda.

\section{Asclepiadaceae.}

† Araujia sericifera, Brot.

$\dagger$ Asclepias curassavica, Linn.

Ceropegia Woodii, Schltr.

Cynanchum acutum, Linn.

$\dagger$ Cryptostegia madagascariensis, Boj.

† Gomphocarpus fruticosus, R. Br.

Hoya carnosa, R. Br. 
Periploca grœca, Linn.

† Stapelia picta, Don.

$\dagger \quad$ tigrina, Decne.

$\uparrow$ Stephanotis floribunda, Brongn

Vincetoxicum officinale, Moench.

\section{Begoniaceae}

Begonia maculata, Raddi.

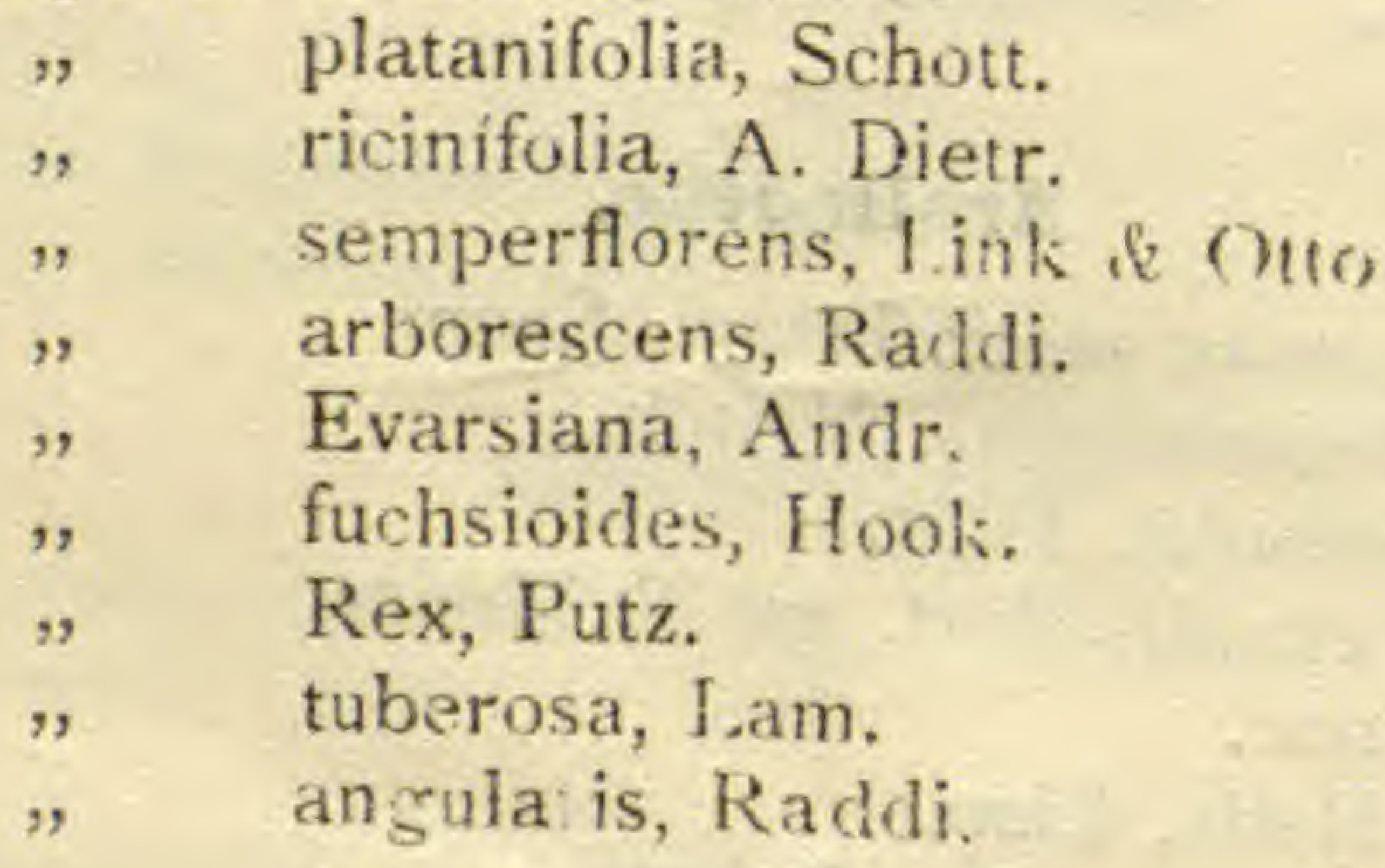

Berberidaceae.

Berberis vulgaris, Linn.

Podophyllum Emodi, Wall.

", peltatum, Linn.

\section{Bignoniaceae.}

$\dagger$ Bignonia Tweediana, Lindl. Eccremocarpus scaber, Ruiz. \& Pav. Jacaranda ovalifolia, R. Br.

$\dagger$ Tecoma capensis, Lindl.
$\dagger "$ jasminoides, Lind. B.R. stans, Juss.

Bixinaceae.

Aberia caffra, Harv, \& Soland. 


\section{Boraginaceae}

$\mathrm{N} \dagger$ Anchusa italica, Retz.

$$
\text { officinalis, Linn. }
$$

$\mathrm{N}+$ Borago officinalis, Linn.

$\mathrm{N}+$ Cerinthe aspera, Roth.

$\mathrm{N}+$ Cynoglossum creticum, Mill. officinale, Linn.

Echium giganteum, Linn.
$\mathrm{N} \dagger \quad " \quad$ italicum, Moench.
$\mathrm{N} \dagger \quad, \quad$ arenarium, Guss.
$\mathrm{N}+\quad$ "plantagineum, Linn.
$\mathrm{N}+\quad$, parviflorum, Moench.

Heliotropium peruvianum, Linn.

$\mathrm{N} \dagger \quad$, europeum, Linn.

$\mathrm{N}$ Lithospermum apulum, Linn.

$\mathrm{N}+$ Myosotis sylvatica, Hoffm.

$\dagger$ Omphalodes linifolia, Moench.

$\mathrm{N}$ Symphytum officinale, Linn.

Tournefortia Messerschmidia, Sweet.

\section{Cactaceae.}

Cereus serpentinus, D.C.

$\begin{array}{ll} & \text { speciosissimus, D.C. } \\ " & \text { rostratus, Linn. } \\ " & \text { Mac Donaldiae, Hook. } \\ " & \text { peruvianus, Mill. } \\ " & \text { Martianus, Zucc. } \\ , \quad & \text { variabilis, Pfeiff. } \\ , \quad \text { triangularis, Mill. } \\ " \quad \text { candicans, Gill. ex Salm. } \\ \text { niger, Salm. }\end{array}$

Echinocactus Decaisnei, Steud.

"

Spec.

Spec.

Epiphyllum truncatum, Haw. Suppl. 
Mamillaria conopsea, Scheidn.

$\begin{array}{ll}, & \text { micromeris, Engelm. } \\ " & \text { magnimamma, Haw. in Till. } \\ " & \text { cespititia, D. C. } \\ \text { spec. } & \text { spec. }\end{array}$

Opuntia andicola, Hort. Angl.

$\mathrm{N} \uparrow \quad$ Amyclaea, Tenore.

Ficus-indica, Lab.

" polyantha, Haw.

, elatior, Mill.

" monacantha, Haw.

" ovata, Pleiff.

" brasiliensis, Haw. Suppl.

, Dillenii, Haw. Suppl.

" microdasys, Pfeiff.

" rufida, Engelm.

" candelabriformis, Hort. Monac.

" cylindrica, D. C.

" leucotricha, D. C.

" tunicata, Haw.

" fragilis, Haw. Suppl.

" Salmiana, Parm. ex Pfeiff.

Pereskia grandifolia, Haw.

Phyllocactus Ackermanni, Walt.

Rhipsalis Cassyta, Gaertn.

" salicornioides, Haw. Suppl.

\section{Campanulaceae.}

$\mathrm{N} \uparrow$ Campanula Erinus, Linn.
$\dagger$
medium, Linn.
$\dagger \quad$,
macrostyla, Boiss \& Helces.
pyramidalis, Linn.
" sulphurea, Boiss. 
Campanula carpatica, Jacq. Hort.

$+\quad$ " $\quad$ Rapunculus, Linn.

" bononiensis, Linn.

" alliariaefolia, Willd.

Canarina campanulata, Linn.

Specularia hybrida, Linn.

$\mathrm{N}$

speculum, D. C.

$\mathrm{N}$ Trachelium coeruleum, Linn.

\section{Capparidaceae.}

$\mathrm{N}+$ Capparis spinosa, Linn. var: rupestris $\mathrm{L}$. Cleome graveolens, Rafin.

\section{Caprifoliaceae.}

$\mathrm{N} \dagger$ Lonicera implexa, Soland. japonica, Thunb.

N Sambucus Ebulus, Linn. nigra, Linn.

Viburnum Opulus, Linn.

, $\quad$ suspensum, Lindl.

"Tinus, Linn.

" tomentosum, Thunb.

\section{Caryophyllaceas.}

$\mathrm{N}$ Alsine procumbens, Vahl.

N " tenuifolia, Linn, Crantz.

$\mathrm{N} \dagger$ Cerastium glomeratum, Thuill.

Cucubalus baccifer, Linn.

† Dianthus Caryophyllus, Linn.

$\dagger \quad, \quad$ chinensis, Linn.

$\dagger \quad$ " Hellwigii, $\times$ Barb. ex Aschers.

" laciniatus, Hort.

" Armeria, Linn.

" gallicus, Pers. 
Dianthus deltoides, Linn. virginicus, Linn.

" barbatus, Linn.

† Gypsophyla elegans, Bieb.

$\dagger$, ", var. rosea, Hort.

$\dagger$ Lychnis coeli-rosea, Desr.

$\dagger \quad$ " coronaria, Desr.

, pyrenaica, Berger.

" Flos-Juvis, Desr. in Lam.

, Flos-Cuculi, Linn.

, segetalis, Hort.

$\mathrm{N}+\quad " \quad$ Githago, Scop.

† Saponaria calabrica, Linn.

$\dagger \quad$ ocymoides, Linn.

$\mathrm{N} \dagger$ Silene nocturna, Linn.

officinalis, Linn.

$\mathrm{N}+\quad$ " nutans, Linn.

" viridifiora, Linn.

$\mathrm{N} \dagger \quad " \quad$ nivea, Muhl. Cat. ex D.C.

$\mathrm{N} \dagger \quad$, inflata, Smith.

$\mathrm{N} \dagger \quad$, italica, Pers.

$N+\quad$, Behen, Linn.

N " " $\quad$ fruticosa, Linn.

$\begin{array}{ccc}\mathrm{N} \dagger & \text { " } & \text { compacta, Fisch. } \\ \mathrm{N} \dagger & \text { sedoides, Poir. } \\ +\quad & \text {, } & \text { var. alba, Hort. }\end{array}$

+ "Drummondi, Hook.

† " aegyptiaca, Linn.

$\dagger$ " pendula, Linn.

$\dagger \quad$ muscipula, Linn.

† " hispida, Desf. 
Silene squamigera, Boiss.

$\mathrm{N}+$ Spergularia rubra Linn.

$\mathrm{N}+$ Stellaria media, Linn.

\section{Casuarinaceae.}

$\dagger$ Casuarina Cunninghamiana, Miq.

, $\quad$ equisetifolia, Linn.
stricta, Dryand. in Ait.

\section{Celastrinaceae.}

Cassine maurocenia, Linn.

$\dagger$ Celastrus punctulatus, Sieb. et Zucc.

Euonymus japonicus, Linn. Fil.

"

"

var. fol. variegatis, Hort.

\section{Chenopodiaceae.}

$\mathrm{N} \dagger$ Atriplex hastatum, Linn.

$\mathrm{N}$ Halimus, Linn.

Beta chilensis, Linn.

$\mathrm{N} \dagger$, maritima, Linn.

$\dagger$ Chenopodium amaranthicolor, Hort.

$\mathrm{N}+$ murale, Linn.

$\mathrm{N}+$

$\mathrm{N}+$ opulifolium Schrad. olidum, Curt.

Hablitzia tamnoides, Bieb.

$\mathrm{N} \dagger$ Kochia tricophylla, Möllers.

$\dagger \quad$, arenaria, Roth. in Schrad.

$\dagger$ Rhagodia nutans. R. Br.

Salicornia herbacea, linn.

$\mathrm{N}+$ Salsola Kali, Linn.

$\mathrm{N} \dagger$ Soda, Linn.

† Spinacia oleracea, Linn. Suaeda fruticosa, Linn. 


\section{Cistaceae.}

N Cistus incanus, Linn.

\section{Compositae.}

$\mathrm{N} \dagger$ Achillea Millefolium, Linn.

$+\quad, \quad$ Ptarmica, Linn.

, magna, Linn.

" santalinoides, Lag. Gen. et Sp.

† , ", Ageratum, Linn.

$\dagger$ " ligustica, All.

† Ageratum brachystephanum, Regel.

$\mathrm{N} \dagger$ Ambrosia maritima, Linn.

$\mathrm{N} \dagger$ Anthemis arvensis, Linn.

$\mathrm{N}$

Urvilleana, D. C.

Triumfetti, All. aitissima, Linn.

Chia, Linn.

austriaca, Jacq.

+ Artemisia arborescens, Linn.

$\dagger \quad, \quad$ Absinthium, Linn.

$\dagger \quad$, Spec.

$\dagger$ Anacyclus Pyrethrum, D. C.

$\dagger$ Ammobium alatum, R. Br.

$\dagger \quad$ ", , grandiflorum, Hort.

$\dagger$ Acroclinum roseum, Hook. Amberboa moschata, Less.

$\dagger$ Aster rotundifolia, Thunb.

" acris, Linn.

† " salicifolius, Ait.

† Dumosus, Linn.

$\mathrm{N} \dagger$ Asteriscus aquaticus, Linn.

$\mathrm{N} \dagger \quad$. $\quad$ spinosus, Linn.

$\mathrm{N} \dagger$ Atractylis cancellata, Linn.

$\mathrm{N} \dagger$ Bellis annua, Linn. 
$\dagger$ Bellis perennis, Linn.

$\mathrm{N}+\quad$, sylvestris, Cyrillo.

$\dagger$ Broteroa trinervata, D. C.

† Barkhausia taraxicifolia, D. C.

$\dagger$ Bidens ferulifolia, D. C.

$\mathrm{N} \dagger$ Calendula arvensis, Linn.

$\mathrm{N}$

fulgida, Raf.

$+\quad, \quad$ officinalis, Linn.

$\dagger$ Callistephus hortensis, Cass.

$\mathrm{N}+$ Carthamus coeruleus, Linn.

$\mathrm{N} \dagger$

$\mathrm{N}+$

$\mathrm{N}+$

lanatus, Linn.

var. tingitanus, Linn.

$\mathrm{N}+$ Catananche lutea, Linn.

Centaurea alpina, Linn.

$\mathrm{N}$

$\mathrm{N}$

$\mathrm{N} \dagger$

$\mathrm{N}+$

+

$\dagger \quad$,

Caleitrapa, Linn.

crassifolia, Bert.

melitensis, Linn.

nicæensis, All.

ragusina, Linn.

Sultani, Hort.

† Calimeris incisa, D. C.

+ Carbenia benedicta, Adans.

$\dagger$ Cacalia articulata, Linn.

$\mathrm{N}$

$\overbrace{}^{+}$

$\mathrm{N}$

$\mathrm{N}+$

$\mathrm{N}+$

$\mathrm{N}+$

$\mathrm{N}$

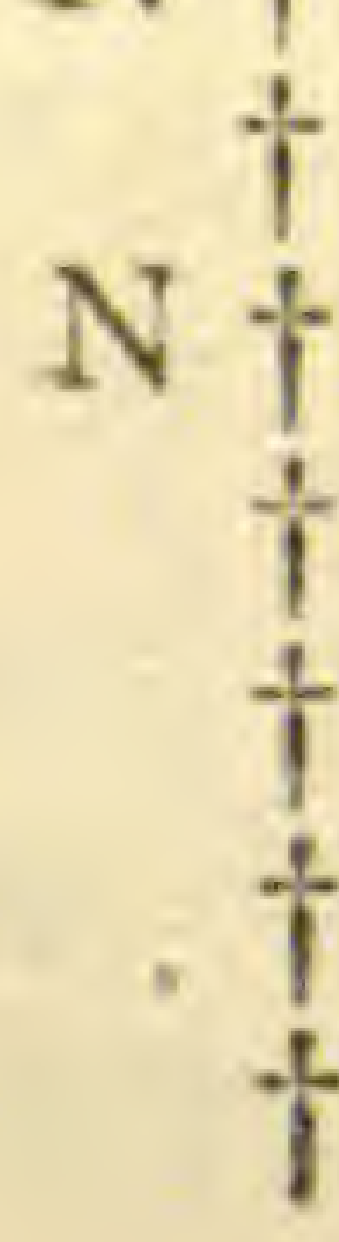

anteuphorbium, Linn.

Carlina lanata, Linn.

involucrata, Poir.

Carduus pycnocephalus, Linn.

marmoratus, Boiss.

Chrysanthemum carinatum, Schoust.

indicum, Linn.

segetum, Linn.

frutescens, Linn.

maximum, Ramond.

"

var. laciniatum, Hort.

Parthenium, Bernh. 
$\dagger$ Cineraria hybrida, Hort. Vilm.

$\dagger$ " , var. compacta, llort.

$\mathrm{N}+$ Cichorium Intybus, Linn.

$\mathrm{N}+\quad, \quad$ pumilum, Jacq.

$\mathrm{N} \dagger$ Conyza ambigua, D. C.

† Coreopsis bicolor, Bosse.

(1)

tr

Tom. Thumb. var. Hort.

" " Drummondi, Torr. \& Gray.

$+\quad, \quad$ tinctoria, Nutt.

$\dagger$ ", hybrida, Hort.

$\mathrm{N}$ Crepis bulbosa, Linn.

$\mathrm{N}+\quad$, virens, $\mathrm{L}$.

† Cosmos bipinnatus, Cav.

$\dagger \quad, \quad$ sulpliureus, Cav.

† Cynara Cardunculus, Linn.

$\dagger$ "Scolymus, Linn.

† Dahlia Juarezi, Hort.

† , variabilis, Desf.

$\uparrow$ Dimorphotheca pluvialis, Moench.

$\dagger$ Dichrocephala latifolia, 1). C.

$\mathrm{N} \dagger$ Evax pygmaea, Linn.

N Echinops Ritro, Linn.

Eupatorium janthinum, Hemsl.

$\dagger$ Erigeron speciosum, D. C.

$\mathrm{N}+$ Filago prostrata, Parl. " spathulata. Presl.

Gazania Pavonia, R Br. in Ait.

$\uparrow$ Galinsoga brachystephana, Regel.

+ Gaillardia pulcheila, Fouger.

† " , var. grandiflora, Hort.

$\mathrm{N}+$ Galactites tomentosa, Moench.

$\dagger$ Helianthus annuus, Linn.

cucumerifolius, Torr. \& Gray.

Helichrysum orientale, Gaertn.

, rupestre, D. C. 
$\mathrm{N} \dagger$ Helminthia echioides, Lini

$\mathrm{N} \dagger$ Hedypnois mauritanica, Schousb.

$\mathrm{N}+$ polymorpha, D. C.

$\mathrm{N} \dagger$ var. tubaeformis, Tenore.

Heliopsis patula, Wendl.

Hieracium vulgatum, Fries.

Helenium quadridentatum, Labill.

$\mathrm{N} \dagger$ Hyoseris lucila, Linn.

$\mathrm{N} \dagger \quad$, radiata, Linn.

$\mathrm{N}+\quad$.. scabra, Linn.

$\mathrm{N} \dagger$ Inula crithmoides, Linn.

$\mathrm{N} \dagger \quad$ graveolens, Linn.

$\mathrm{N} \uparrow, \quad$ viscosa, Linn.

$\mathrm{N}+$ Jasonia glutinosa, Linn.

Kleinia articulata, Haw.

$\uparrow$ Lactuca sativa, Linn.

$\mathrm{N}+$ virosa, Linn.

Lappa major, Gaertn.

Lapsana communis, Linn.

Lonas inodora, Gaertn.

$\dagger$ Jeptusyne maritima, A. Gray in Proc.

$\mathrm{N} \dagger$ Maruta cotula, D. C.

$\mathrm{N} \dagger$ Melitella pusilla, Somm.

$\mathrm{N} \dagger$
Mi"
" var. laciniata, Borg.

Mikania scandens, Willd.

$\mathrm{N} \dagger$ Matricaria Chamomilla, Linn.

$\dagger \quad, \quad$ inodora, Linn.

Montanoa bipinnatifida, C. Kuch.

Madia sativa, Molina. Sagg.

$\mathrm{N} \dagger$ Notobasis syriaca, Linn.

$\mathrm{N} \dagger$ Onopordon Sibthorpianum, Boiss.

† Palafoxia texana, D. C.

i Podolepis acuminata, R. Br. in Ait.

$\mathrm{N} \dagger$ Picridium vulgare, Desf.

N Podospermum lacinatum, Linn.

$\mathrm{N} \dagger$ Phagnalon graecuna, Boiss. 
N Pulicaria dysenterica, Linn.

Pyrethrum cinerariaefolium, Trev.

$\dagger$ hybridum, Wendel.

" Parthenium, Sm. Fl. Brit.

$\mathrm{N}+$ Rhagadiolus stellatus, Linn.

Rudbeckia amplexicaulis, Vahl. in Skriv.

Rodigia commutata, Spreng.

$\mathrm{N}_{\text {st }}$ Senecio Cineraria, D. C.

$\dagger \quad " \quad$ Petasites, D. C.

$\begin{array}{lll}\mathrm{N} \dagger & \quad & \text { pygmeus, D. C. } \\ \mathrm{N} \dagger \quad & \quad \text { leucanthemifolius, Poir. }\end{array}$

$\mathrm{N}+\quad$ " Koempferi, D. C.

+ " vulgaris, Linn.

" elegans, Linn.

" umbrosus, Waldst.

" Doria, Linn.

", erucifolius, Eenth.

$\mathrm{N} \dagger$ Scolymus hispanicus, Linn.

$\mathrm{N}$

grandiflorus, Desf.

Solidago fragrans, Willd.

† Spilanthes Acmella, Murr. Syst.

$\mathrm{N}+$ Silybum marianum, Linn.

Santolina chamaecyparissus, Linn.

$\mathrm{N}+$ Son, viridis, Willd.

Sonchus levis, Bert.

$\mathrm{N} \dagger \quad$, asper, Hill.

$\mathrm{N} \dagger \quad$ " tenerrimus, Linn.

$\dagger$ Stevia spec.

$\dagger$ Tagetes erecta, Linn.

$\dagger \quad$, minuta, Linn.

$\dagger \quad$ patula, Linn.

† " " var, bicolor, Hort.

$\dagger$, signata pumila, Bartl.

Tanacetum boreale, Fisch. ex Link.

N Thrincia tuberosa, Linn. 
Tragopogon pratense, Linn.

$\mathrm{N} \dagger$ Urospermum picroides, Linn.

Verbesina alata, Linn.

$\mathrm{N} \uparrow$ Xanthium spinosum, Linn.

strumarium, Linn.

$\dagger$ Xeranthemum annuum, Linn.

† " " var. superbissimum, Hort.

$\dagger$ Zinnia elegans, Jacq.

$\dagger$," grandiflora, Nutt. in Trans.

Araucaria Bidwilli, Hook.

\section{Coniferae.}

" Brasiliana, A. Rich.

" Cooki, R. Br. ex Don.

$\mathrm{N} \dagger$ Callitris quadrivalvis, Vent.

Cupressus macrocarpa, Hartw.

‘ elegans, Hort.

$\dagger \quad " \quad$ sempervirens, Linn, var: horizontalis Hort.

$\dagger \quad$ „ sempervirens, Linn. var: pyramidalis

Hort.

$\dagger \quad$ " sempervirens, L.inn. var: fastigiata Hort.

Ginkgo biloba, Linn.

$\mathrm{N}+$ Pinus halepensis, Mill.

$\dagger$, Pinaster, Soland.

Podocarpus macrophylia, D. Don. in Lamb.

$\dagger$ Thuya occidentalis, Linn.

$\dagger \quad$ orientalis, Linn.

\section{Convolvulaceae.}

$\mathrm{N} \dagger$ Convolvulus althaeoides, Linn.

pentapetaloides, Linn.

Sepium, Linn. 
$\mathrm{N}+$ Convolvulus tricolor, Linn.

$\mathrm{N}+$

$\mathrm{N}$

$\dagger \quad$, $\quad$ farinosus, Linn.

$\dagger$ Ipomoea rubro-roerulea, Hook.

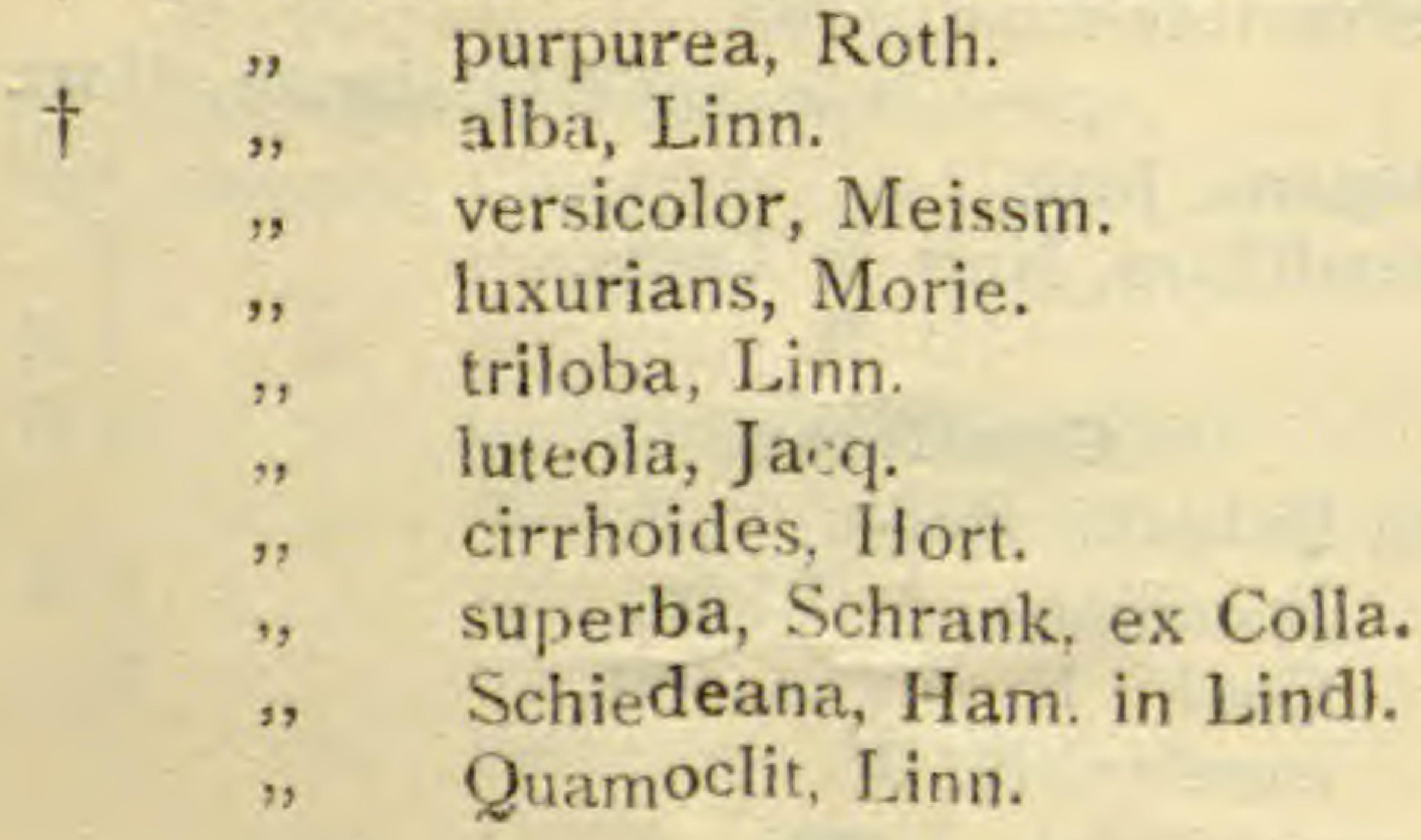

\section{Cornaceae.}

Aucuba japonica, Thunb. Garrya elliptica, Dougl.

\section{Crassulaceae,}

Bryophyllum calycinum, Salisb.

Cotyledon orbiculata, Linn.

Crassula crenata, Desf. perfossa, Lam.

" lactea, Lam.

, coccinea, Linn.

" lycopodioides, Lam.

") spathulata, Thunb.

† Kalanchoë thyrsiflorum, Haw. in Harv. C. Soland. Rochea falcata, D. C. Sedum dasyphyllum, Linn.

" heptapetalum, Linn.

" nicaense, All.

$\mathrm{N} \dagger \quad$ " rubens, Linn. 
$\mathrm{N} \dagger$ Sedum stellatum, Linn.

$\mathrm{N}$

Telephium, Linn.

N Sempervivum arboreum, Linn.

$\mathrm{N} \dagger$ Umbilicus horizontalis, Guss.

N Tillaea muscosa, Linn.

\section{Cruciferae.}

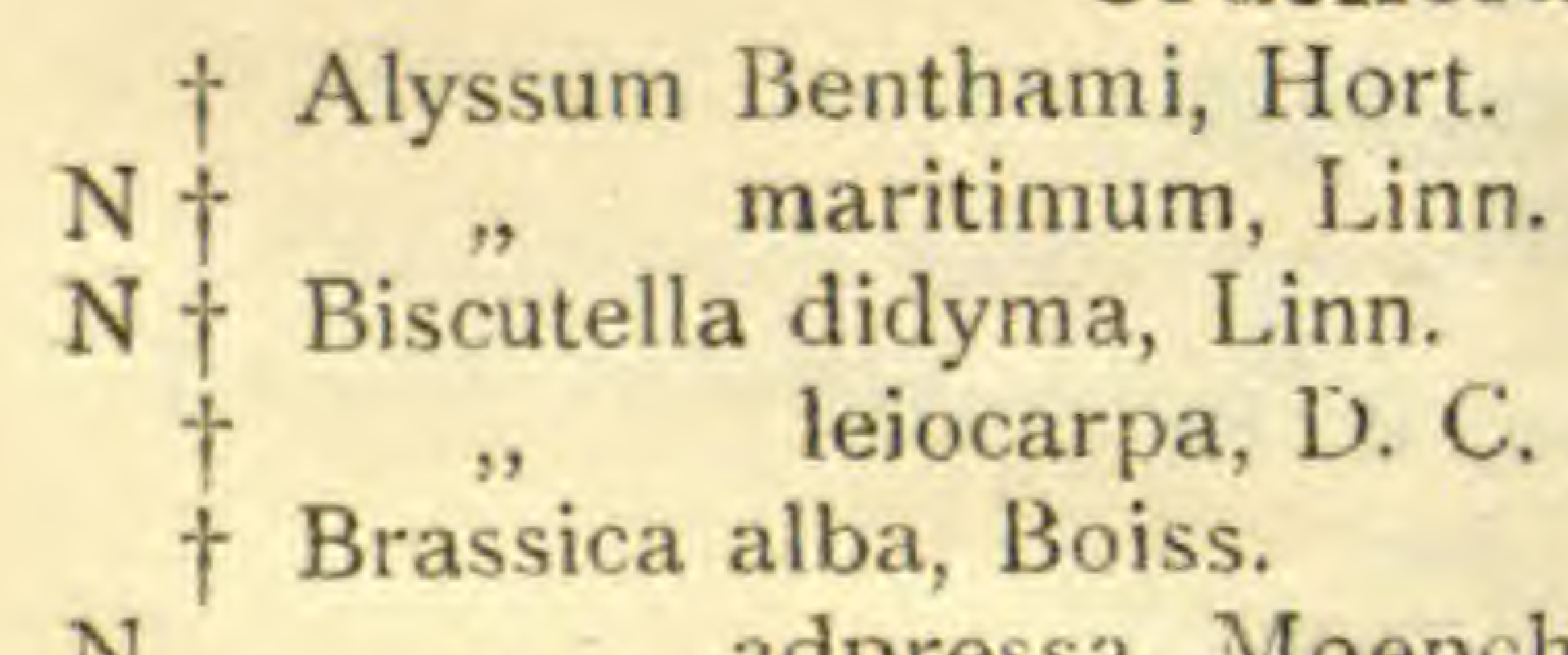

$\mathrm{N}$ adpressa, Moench.

$\mathrm{N} \dagger \quad$ " campestris, Linn.

$\mathrm{N} \dagger \quad, \quad$ nigra, Kock.

$\dagger \quad " \quad$ oleracea, Linn.

$\mathrm{N} \dagger \quad$, Sinapistrum, Boiss.

$\mathrm{N} \dagger$ Cakile maritima, Scop.

$\mathrm{N} \dagger$ Capsella Bursa-pastoris, Linn.

† Camelina sativa. Crantz, Stirp.

$\mathrm{N} \dagger$ Cheirantkus Cheiri, Linn.

$\mathrm{N} \dagger$ Conringia orientalis, Dum.

Cochlearia Armoracia, Linn.

$\mathrm{N} \dagger$ Coronopus didymus, Linn.

$\mathrm{N}+$

$\mathrm{N} \dagger$ Diplotaxis erucoides, D. C.

$\mathrm{N} \dagger$

$\mathrm{N}+$

$\mathrm{N}+$

procumbens, Gilibert.

$\mathrm{N} \dagger$ Eruca sativa, Mill.

N Enarthrocarpus pterocarpus, Pers.

$\dagger$ Erysimum officinale, Linn.

Hesperis matronalis, Linn。

† Iberis amara, Linn.

" Gibraltarica, Linn. 
† Iberis sempervirens, Linn.

$\dagger$, umbellata, Linn.

† Ionopsidium acaule, Reicht.

Isatis tinctoria, Linn.

$\mathrm{N}+$ Lepidium Draba, Linn.

$\mathrm{N} \dagger \quad$, lberis, Linn.

$\dagger \quad$ sativum, Linn.

† , virginicum, Linn.

t Lunaria annua, Linn.

$\mathrm{N} \dagger$ Malcolmia africana, $\mathrm{R} . \mathrm{Br}$.

$\mathrm{N}+$, maritima, R. Br.

$\mathrm{N}+$ Matthiola incana, Linn.

$\begin{array}{rrr}\dagger & \text { " } & \text { " var. alba, } \\ \dagger & " & \\ \mathrm{~N} \dagger & \text { " var. azurea, }\end{array}$

$\mathrm{N}+$ Nasturtium officinale, R. Br.

$\mathrm{N}+$ Raphanus Landra, Moretti.

$\mathrm{N} \dagger$ Sisymbrium altissimum, Linn.

Raphanistrum, Linn.

$\mathrm{N}+$

$\mathrm{N}+$

Irio, Linn.

" officinale, I inn.

† Succovia balearica, Merlik.

Cucurbitaceae.

† Bryonopsis laciniosa, Naud.

Benincasa cerifera, Savi.

† Citrullus vulgaris, Schrad.

† Cucumis melo, Linn.

$\dagger$ Cucurbita pileiformis. M. Roem.

$\mathrm{N} \dagger$ Ecballium Elaterium, A. Rich.

† Lagenaria vulgaris, Ser.

$\dagger$ Luffa acutangula, Roxb.

" spec.

$\dagger$ Momordica Charantia, Linn. 
Sechium edule, Sweet.

Trichosanthes anguinea, Linn.

Corylus Avellana, Linn.

Quercus Ilex, Linn.

$\dagger$ " Robur, Linn var: pedicellata.

\section{Cupuliferae}

\section{Cycadere.}

Ceratozamia mexicana, Brongn.

+ Cycas revoluta, Thumb.

\section{Dipsaceae,}

$\mathrm{N} \dagger$ Scabiosa atropurpurea, Linn.

$+\quad$, maritima, Linn.

\section{Elatinaceae.}

N Elatine Hydropiper, Linn, var macropoda, Guss.

\section{Ericaceae.}

N Erica multiflora, Linn.

\section{Euphorbiaceae.}

Acalypha cuneata, Poepp.

" musaica, Hort.

N Andrachne telephioides, Linn.

Buxus balearica, Lam.

, sempervirens, Linn.

$\mathrm{N}+$ Crozophora tinctoria, Linn.

$\mathrm{N}+$ Euphorbia aleppica, Linn.

$\begin{array}{cll} & " & \text { altissima, Boiss. } \\ \mathrm{N}+ & " & \text { pinea, Linn. } \\ \mathrm{N}+ & " & \text { Ipecacuhana, Linn. }\end{array}$


† Euphorbia platiphylla, Linn.

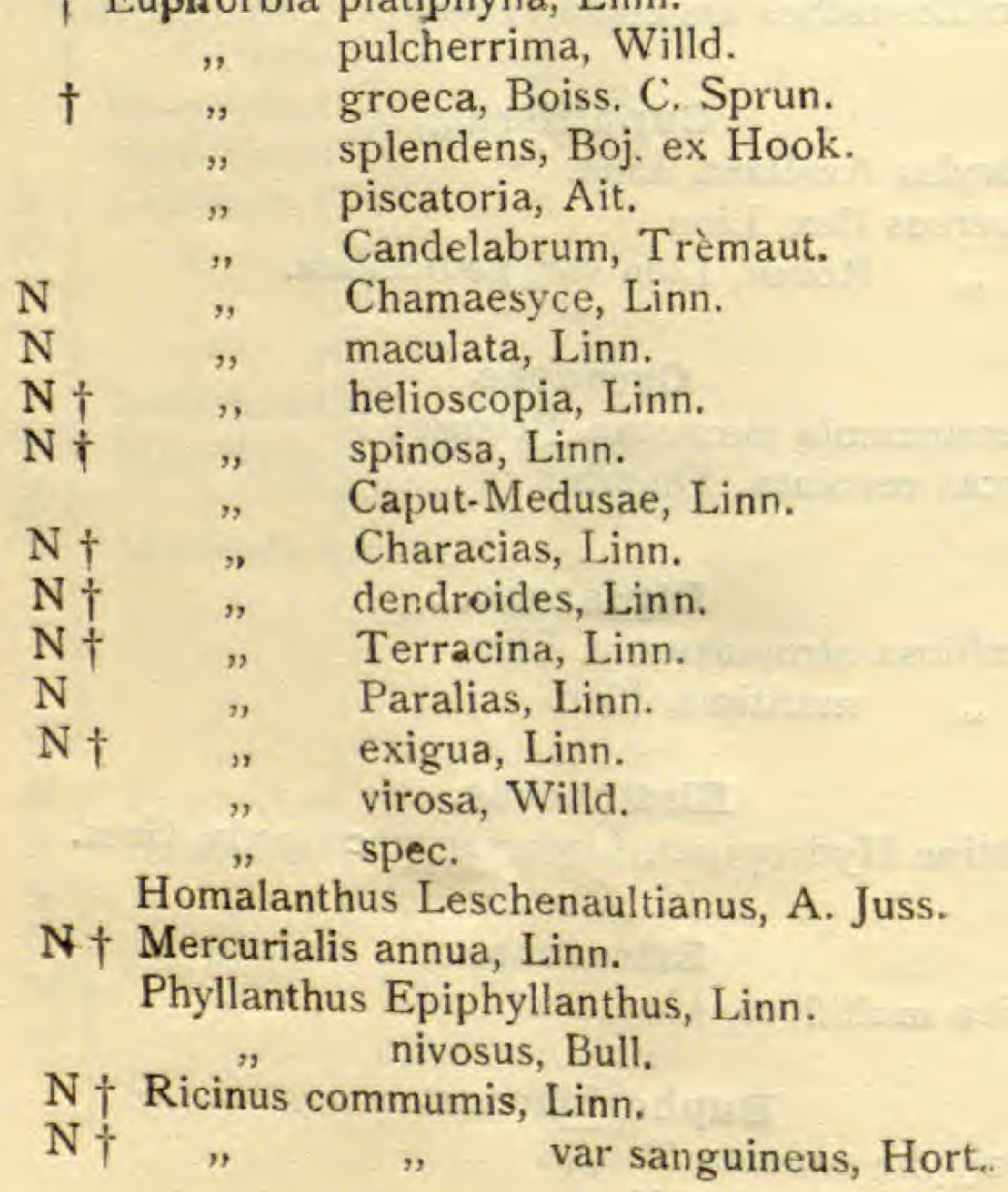

\section{Ficoideae.}

N Mesembryanthemum acinaciforme, Linn.

\begin{tabular}{|c|c|c|}
\hline \multirow{3}{*}{$\mathrm{N} \dagger$} & ", & ciliatum, Thunb. \\
\hline & ", & $\begin{array}{l}\text { cordifolium, Linn, } \\
\text { crystallinum. Linn. }\end{array}$ \\
\hline & $"$ & $\begin{array}{l}\text { coccineum, Haw. } \\
\text { deltoides, Linn. }\end{array}$ \\
\hline $\mathrm{N} \dagger$ & $"$ & nodiflorum, Linn. \\
\hline & $"$ & rubricaule, Willd, \\
\hline
\end{tabular}


Mesembryanthemum uncinatum, Linn.

$\dagger$ Tetragonia expansa Murr. in Comm.

\section{Frankeniaceae.}

$\mathrm{N}$ Frankenia intermedia, D. C.

$\mathrm{N}$ pulverulenta, Linn,

Fumariaceae.

$\mathrm{N} \dagger$ Fumaria agraria, Leg.

$\begin{array}{lll}\mathrm{N} \dagger & \quad \text { densiflora, U. C. } \\ \mathrm{N} \dagger \quad & \text { officinalis, Linn. }\end{array}$

Geraniaceae.

$\mathrm{N} \dagger$ Erodium cicutarium, Linn.

$\mathrm{N} \dagger \quad, \quad$ laciniatum, Cav.

$\mathrm{N}+\quad " \quad$ malachoides, Linn.

$\mathrm{N} \dagger$ " moschatum, Burn.

$\mathrm{N} \dagger$ Geranium dissectum, Linn.

$\mathrm{N} \dagger \quad, \quad$ grandiflorum, Willd.

N " anemonaefolium, L'Her.

$\mathrm{N} \dagger \quad, \quad$ molle, Linn.

$\mathrm{N} \dagger \quad, \quad$ Robertianum, Linn.

$\mathrm{N} \dagger \quad, \quad$ rotundifolium, Linn.

$\dagger$ Impatiens Balsamina, Linn.

Sultani, Hook. f.

† Pelargonium graveolens, L'Her.

$\dagger \quad$ " hybridum, Soland.

$\mathrm{N} \dagger \quad, \quad$ inquinans, Soland. in Aiz.

odoratissimum, Soland.

". triste, Soland.

$\dagger \quad$ ", zonale, L'Her. ex Soland.

$\dagger$ Tropaeolum majus, Linn.

" $\quad$ Tom Thumb var. Hort.

". peregrinum, Linn. 
Gentianeae.

$\mathrm{N}+$ Chlora perfoliata, Linn.

$\mathrm{N}+$ Erythrea pulchella, Sw.

, Centaurium, Pers.

Gesneraceae.

Tydaea picta, Dcne.

Hydrophylleae.

$\dagger$ Nemesia insignis, Bentn.

† Phacelia campanularia, A. Grag.

Hypericineae.

N Hypericum aegyptiacum, Linn.

$\begin{array}{lll}\mathrm{N} & \prime & \text { chinense, Linn. } \\ \mathrm{N} & \prime & \text { crispum, Linn. }\end{array}$

$\mathrm{N} \quad$ "tomentosum, Linn.

Illecebraceae.

$\mathrm{N}+$ Herniaria cinerea, Linn.

$\mathrm{N}+$ Paronychia argentea, Linn.

$\mathrm{N} \dagger \quad$ nivea, D. C.

$\mathrm{N} \dagger$ Polycarpon tetraphyllum, Linn.

Juglans regia, Linn.

\section{Juglandaceae.}

Labiatae.

$\mathrm{N} \dagger$ Ajuga Iva, Linn.

$\mathrm{N}$

" reptans, Linn.

$\mathrm{N} \dagger$ Ballota nigra, Liun.

† Coleus Verschaffeltii, Ch. Lem.

$\dagger$ Hyssopus officinalis, Linn.

$\mathrm{N} \dagger$ Lamium amplexicaule, Linn. 
† Lavandula dentata, Linn.

$\dagger \quad$, multifida, Linn.

$\dagger \quad, \quad$ Spica, Cav.

† Leonotus Leonurus, R. Br.

Lophanthus anisatus, Benth.

$\mathrm{N}+$ Marrubium vulgare, Linn.

$\mathrm{N}+$ Melissa officinalis, Linn.

$\mathrm{N} \dagger$ Mentha aquatica, Linn.

$\begin{array}{lll}\dagger & \quad \text { piperita, Linn. } \\ & \text { " } & \text { Pulegium, Linn. }\end{array}$

$\dagger \quad$ viridis, Linn.

Nepeta latifolia, D. C.

$\dagger$ Ocimum Basilicum, Linn.

$\dagger \quad$ " minimum, Linn.

† Origanum majorana, Linn.

vulgare, Linn.

$\mathrm{N} \dagger$ Phlomis fruticosa, Linn.

$\mathrm{N} \dagger$ Prasium majus, Linn.

N Rosmarinus officinalis, Linn.

$\mathrm{N}$

$\dagger$ Satureja hortensis, Jinn. var. prostratus, Hort.

$\mathrm{N}+$

$$
\text { " microphylla, D'Urville. }
$$

$\mathrm{N}+$, Nepeta, Linn.

Salvia argentea, Linn.

, azurea, Lam.

$\mathrm{N}+\quad$ " coccinea, Juss, et Musr.

$\mathrm{N} \dagger \quad$ " clandestina, Linn.

" Grahami, Benth.

$\mathrm{N}+\quad$ " involucrata, Cav.

$\mathrm{N} † \quad " \quad$ triloba, Linn.

$\mathrm{N} \dagger$ Sideritis romana, Linn.

$\mathrm{N} \dagger$ Stachys hirta, Linn.

$\mathrm{N}+$ Thymus capitatus, Linn.

", vulgaris, Linn. 
N Teucrium Chamoedrys, Linn.

$\mathrm{N} \dagger \quad$ " flavum, Linn.

$\mathrm{N} \dagger \quad$, fruticans, Linn.

" Marum, Linn.

Lauraceae.

$\mathrm{N} \uparrow$ Laurus nobilis, Linn.

Ocotea foetens, Benth C. Hooker.

Persea gratissima, Gaertn.

Leguminosae.

$\dagger$ Acacia cyanophylla, Lindl.

$\dagger$ " Farnesiana, Willd.

+ $\quad$ longifolia, Willd.

$\dagger \quad$ spadicigera, Cham.

$\dagger$ " calamifolia, Sweet.

† " lophanta, Willd.

$\dagger \quad$ " armata, R. Br.

" glaucescens, Willd.

" penninervis, Sieber.

$" \quad$ pentadenia, Lindl.

"uncinella, Benth.

$\dagger$ Albizzia Julibrissim, Durazzini.

$\mathrm{N} \dagger$ Anagyris foetida, Linn.

$\dagger$ Arachys hypogea, Linn.

$\mathrm{N}$

Anthyllis Hermanniae, Linn.

$\mathrm{N}$

$\mathrm{N} \dagger$ tetraphylla, Linn. Vulneraria, Linn.

Amorpha fruticosa, Linn.

$\mathrm{N} \dagger$ Astragalus boeticus, Linn.

$\mathrm{N}+$

"

hamosus, Linn.

sesameus, Linn.

Bauhinia purpurea, Linn.

† Caesalpinia Gillesii, Wall, ex Hook. 
† Cassia enarginata, Linn.

$\dagger \quad$ laevigata, Willd. " fistula, Linn.

$\dagger$ " floribunda, Cav.

$\mathrm{N}+$ Ceratonia Siliqua, Linn.

$\dagger$ Cercis Siliquastrum, Linn.

$\dagger \quad$, canadensis, Linn.

$\uparrow$ Cicer arietinum, Linn.

$\mathrm{N}+$ Coronilla Emerus, Linn.

$\mathrm{N}+\quad, \quad$ scorpioides, Linn.

$\mathrm{N} \dagger \quad$ Valentina, Linn.

† Cytisus albus, Link.

Dioclea glycinoides, Hort.

Desmodium pendulum, F. Muel.

$\mathrm{N} \dagger$ Dolichos Lablab, Linn.

$\mathrm{N} \dagger$ Ervum Ervilia, Linn.

$\mathrm{N} \dagger \quad$ "gracile, Lois.

$\mathrm{N}+\quad$ Lens, Linn.

Galega officinalis, Linn.

Genista aethnensis, D. C.

$\dagger \quad$ " Alba, Linn.

Gleditschia caspica, Desf.

$\dagger$, triacanthos, Linn.

Glycyrr hiza glabra, Linn.

$\mathrm{N} \dagger$ Hedysarum coronarium, Linn.

$\mathrm{N} \dagger$ Hippocrepis ciliata, Willd.

multisiliquosa, Linn.

$\mathrm{N}+$ Hymenocarpus circinatus, Savi.

Indigofera australis, Willd.

Kennedya nigricans, Lindl.

$\mathrm{N} \dagger$ Lathyrus Aphaca, Linn. 
$\mathrm{N}$ †管Lathyrus Clymenum, Linn.

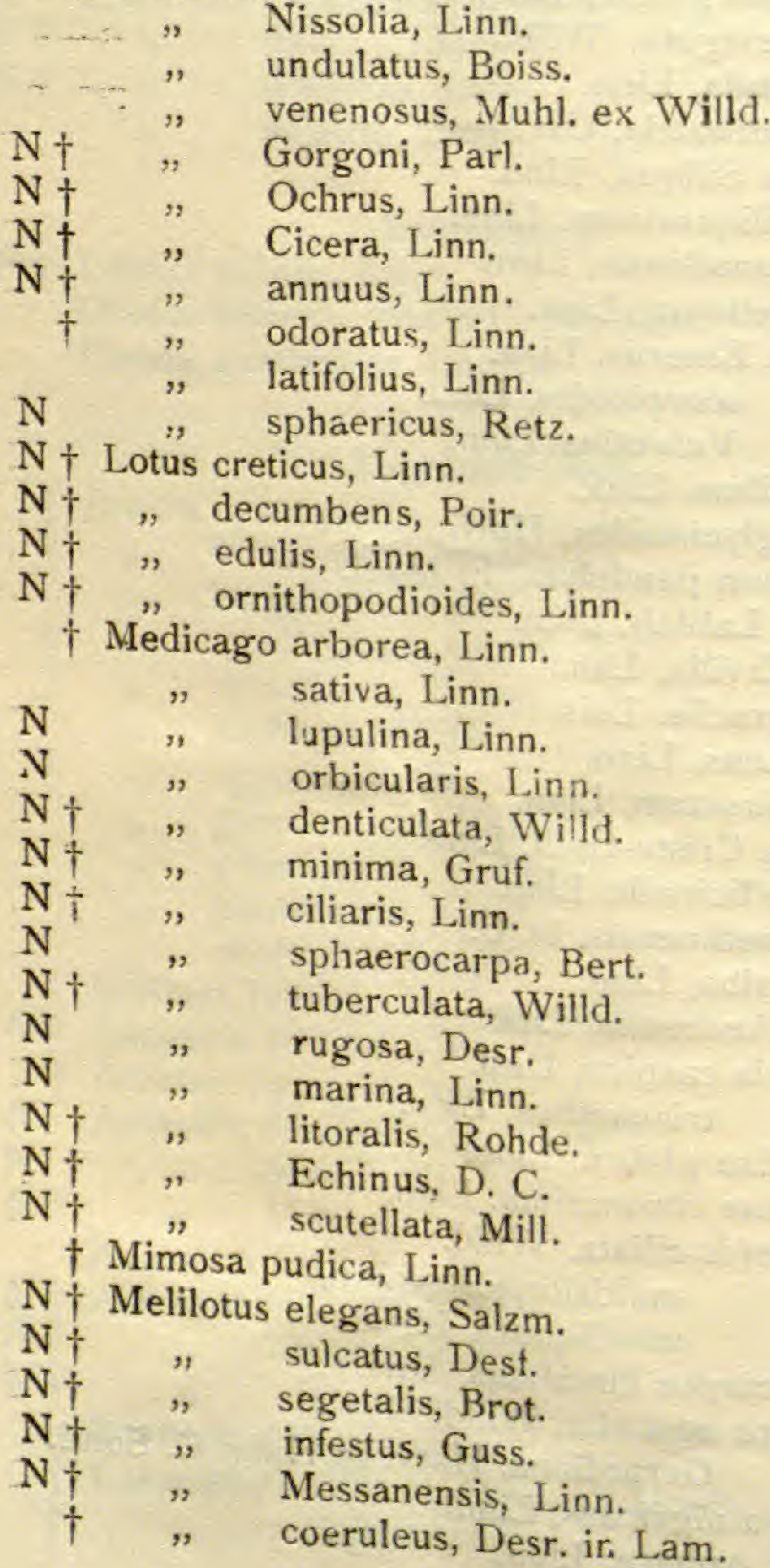


Myroxylon Pereirae, Klotzseh.

$\mathrm{N}$ Ononis reclinata. Linn.

$\mathrm{N}+\quad, \quad$ ornithopodioides, Linn.

$\mathrm{N} \dagger \quad$, Sicberi; Besser.

$\mathrm{N}$

Natrix, Linn.

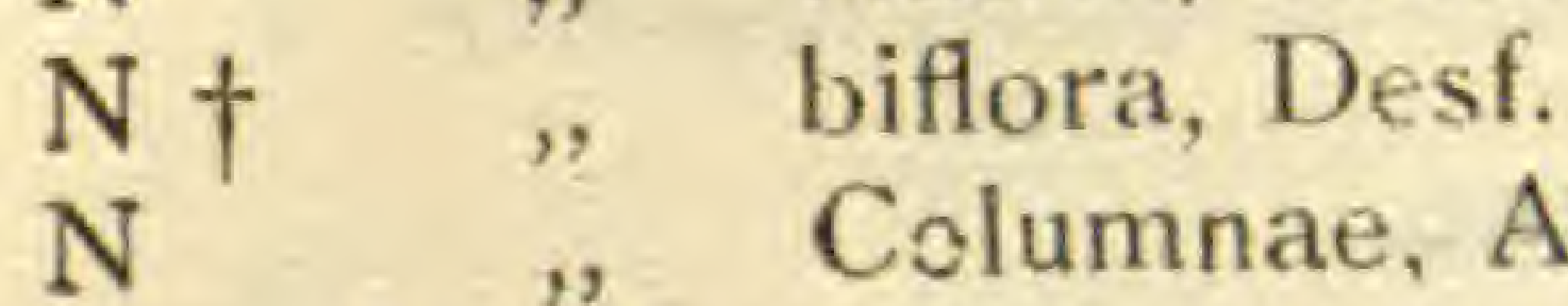

Columnae, All.

† Parkinsonia aculeata, Linn.

$\dagger$ Phaseolus Caracalla, Linn.

$\mathrm{N}+$ Pisum arverse, Linn

Poinciana regia, Boj.

$\mathrm{N} \dagger$ Psoralea bituminosa, Linn.

Schotia brachypetala, Sond.

$\mathrm{N}+$ Scorpiurus subvillosa, Linn.

$\uparrow$ Sophora japonica, Linn.

$\mathrm{N}+$ Spartium junceum, Linn.

Swainsonia rosea grandiflora, Hort.

Tamarindus indica, Linn.

Templetonia retusa, $\mathrm{R}$. Br.

$\mathrm{N} \dagger$ Tetragonolobus purpureus, Linn.

$\mathrm{N}+$ Trifolium stellatum, Linn.

$\mathrm{N} \dagger$

$\mathrm{N}+\quad+$

$\mathrm{N}+\quad$,

$\mathrm{N} \dagger$

$\mathrm{N}$

$\mathrm{N}+\quad$ "

$\mathrm{Ni} \quad$ "

$\mathrm{N} \dagger \quad$,

$\mathrm{N}+\quad$,

$\mathrm{N}+\quad$,

$\mathrm{N}$

$\mathrm{N}+$ Vicia lutea, Linn.

† Trigonella Foenum-groecum, Linn.

$\dagger \quad$ Faba, Linn. scabrum, Linn.

resupinatum, Linn.

tomentosum, Linn.

suffocatum, Linn.

repens, Linn.

var. hibernicum, Hort.

nigrescens, Viv.

agrarium, Linn.

intermedium, Guss.

angustifolium, Linn. 
$\mathrm{N} \dagger$ Vicia narbonensis, Linn.

$\mathrm{N} \dagger \quad " \quad$ " var serratifolia, Hort.

$\mathrm{N} \dagger \quad$, sativa, Linn.

$\mathrm{N} \quad, \quad$, var. amphicarpa, Hort.

$\mathrm{N} \dagger \quad$ villosa, Roth.

\section{Linaceae.}

$\dagger$ Linum africanum, Linn.

$\dagger \quad$ " campanulatum, Linn.

$\mathrm{N} \dagger \quad$ " decumbens, Desf.

$\dagger$ " perenne, Linn.

$\dagger \quad$ regale, Hort. Van Houtte.

$\dagger \quad$ strictum, Linn.

$\dagger \quad$ usitatissimum, Linn.

$\dagger \quad$ "grandiflorum, Desf.

$\mathrm{N} \dagger \quad$, gallicum, Linn.

$\dagger$ Radiola linoides, Roth.

\section{Lobeliaceae.}

$\dagger$ Lobelia Erinus, Linn.

† " " " $\quad$ " var, alba, Hort.

\section{Lythraceae.}

$\mathrm{N} \dagger$ Lythrum Graefferi, Ten.

$\mathrm{N} \dagger \quad$ " hyssopifolia, Linn.

$\dagger \quad$ Salicaria, Linn.

N Punica Granatum, Linn.

,

var. fl. pleno, Hort.

\section{Magnoliaceae:}

Magnolia grandiflora, Linn.

\section{Malpighiaceae.}

Malpighia glabra, Linn。 


\section{Malvaceae.}

$\mathrm{N}+$ Abutilon Avicennae, Gaertn.

$\dagger \quad " \quad$ Bedfordianum, A. St. Hill.

" incanum, Sweet.

" striatuni, Dicks.

† Althaea ficifolia, Cav.

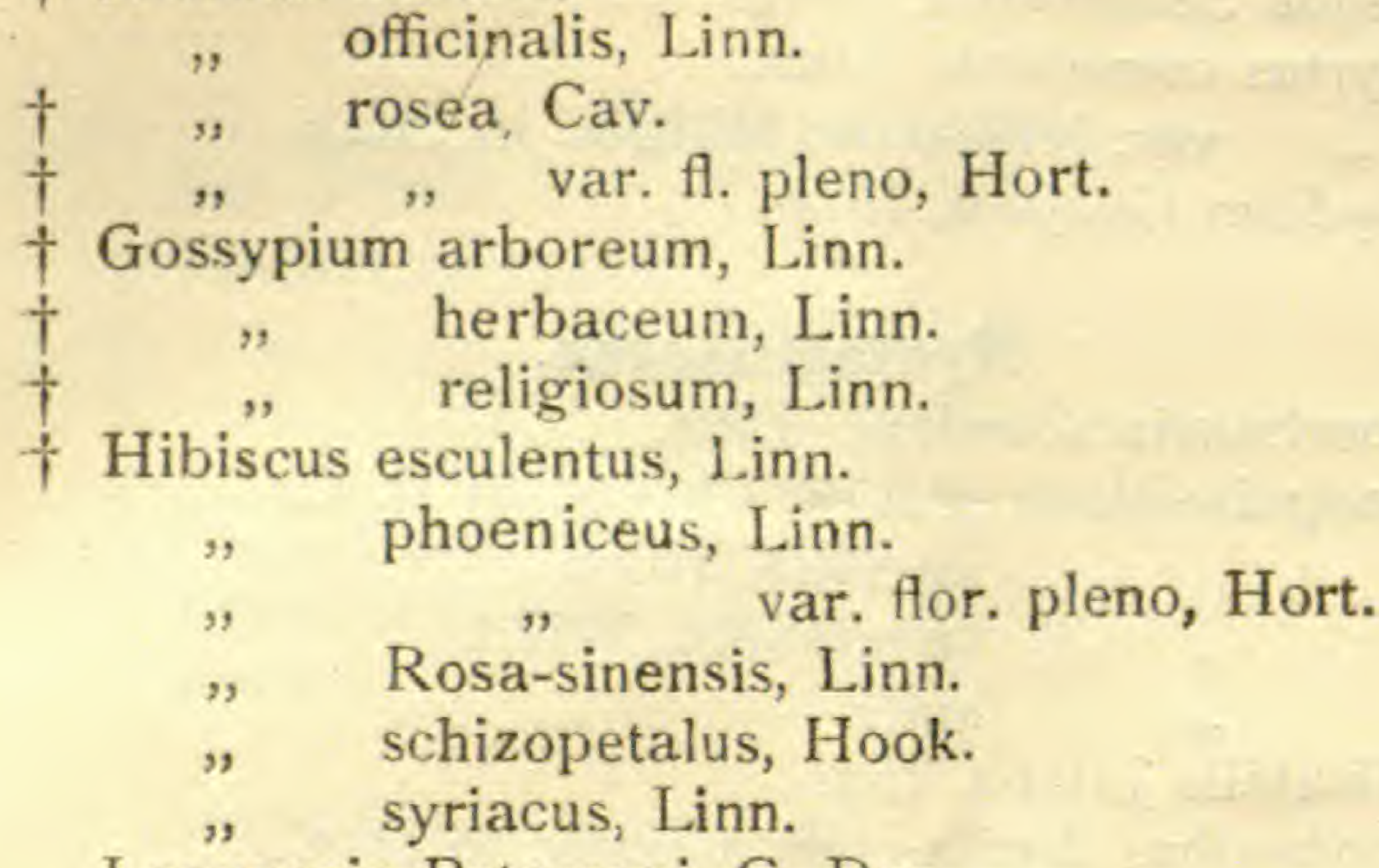

Lagunaria Patersoni, G. Don.

$\mathrm{N} \dagger$ Lavatera arborea, Linn.

$\mathrm{N}+\quad " \quad$ cretica. Linn.

$\mathrm{N} \dagger \quad, \quad$ trimestris, Linn.

$\dagger$ Malope trifida, Cav.

$\dagger$, moschata, Linn.

$\mathrm{N} \dagger \quad, \quad$ sylvestris, Linn. var. alba, Hort.

Malvaviscus arboreus, Cav.

† Pavonia hastata, Cav.

Meliaceae.

† Melia Azedarach, Linn.

Myoporineae.

$\mathrm{N} \dagger$ Myoporum serratum, $\mathrm{R}$. Br. 


\section{Myrtaceae.}

$\dagger$ Eucalyptus corynocalyx, F. Muel.

globulus, Labill.

" longifolia, Link.

Eugenia Smithii, Poir.

Feijoa Sellowiana, Berg.

$\mathrm{N} \dagger$ Myrtus communis, Linn.

$\dagger$ " var: leiocarpa, Wright. in Sauv.

Psydium Guajava, Linn.

\section{Nyctaginaceae.}

Boerhaavia scandens, Linn.

Bougainvillaea glabra, Choisy. in D. C.

lateritia, Hort.

,

"

spectabilis, Willı.

spec.

$\mathrm{N} \dagger$ Mirabilis Jalapa, Linn.

Oxybaphus floribundus, Choisy. in D. C.

Nymphaeaceae.

Nelumbium speciosum, Willd.

Numphar luteum, Sibth. C. Sm.

Nymphaea alba, Linn.

" rosea, Sweet.

\section{Oleaceae.}

$\dagger$ Jasminum azoricum, Linn.

" fruticans, Linn.

" grandiflorum, Linn.

" officinale, Linn.

" primulinum, Hort.

" Sambac, Soland. in Ait.

$\dagger$

Ligustrum japonicum, Thumb.

"nepalense, Wall.

" ovalifolium, Hassk. 
Ligustrum pyrenaicum, Gouan.

Linociera compacta, R. Br.

$\mathrm{N} \uparrow$ Olea europraea, Linn.

$\dagger$ " " var, sativa, H. et $\mathrm{L}$.

Osmanthus fragrans, Lour.

Syringa vulgaris, Linn.

\section{Onagrariaceae.}

Roisduvalia Douglasii, Spach.

$\dagger$ Clarkia alba, Hort.

$\dagger \quad$ " " var. fl. pl., Hort.

† , elegans, Dougl.

$\dagger \quad, \quad$ integrifolia, Hort.

$\dagger \quad, \quad$ pulchella, Pursh.

$\mathrm{N} \uparrow$ Epilobium Tournefortii, Michal.

Fuchsia coccinea, Soland. in Ait.

macrostemma, Ruitz. C. Pav.

Lopezia racemosa, Cav.

Oenothera amoena, Lehm.

$\dagger$

biennis, Linn.

" gloriosa, Hort.

rosea, Soland. in ait.

† ". $\quad$ Whamineyi, A. Gray, in Proc.

\section{Oxalidaceae.}

N Oxalis cernua, Thumb. (bulbilli).

$\mathrm{N}$

$\mathrm{N}+\quad " \quad$ corniculata, Linn.

" purpurea, Linn.

\section{Papaveraceae.}

$\dagger$ Argemone Barkleyana, Penny.

Bocconia cordata, Willd.

$\mathrm{N}_{\dagger}$ Chelidonium majus, Linn. 
Corydalis glauca, Pursh.

$\dagger$ Eschscholtzia californica, Cham.

$\dagger \quad, \quad$ " crocea slba, Benth.

$\mathrm{N} \dagger$ Glaucium flavum, Crantz.

Hunnemannia fumariaefolia, Sweet.

$\dagger$ Hypecoum procumbens, Linn.

Meconopsis cambrica, Vig.

Papaver bracteatum, Lindl.

\begin{tabular}{|c|c|c|}
\hline $\mathrm{N} \dagger$ & " & $\begin{array}{l}\text { hybridum, nudicaule, Lin } \text {. } \\
\text { nudicaule. Linn }\end{array}$ \\
\hline & & pinnatifidum, Moris. \\
\hline & " & Rhoeas, Linn. \\
\hline & $"$ & setigerum, D. C. \\
\hline & $"$ & somniferum, Linn. \\
\hline & " & \\
\hline
\end{tabular}

Passifloreae.

Passiflora alba, Link \& Otto.

$" \quad$ coccirea, Aubl.

" coerulea, 1 inn.

Pedalineae.

$\dagger$ Sesamum indicum, Linn.

\section{Phytolaccaceae.}

Phytolacca decandra, Linn. $\dagger$ dioica, Linn.

$\dagger$ Rivina humilis, Linn.

\section{Piperaceae.}

Piper nigrum, Linn. 


\section{$-35 \cdots$}

\section{Pittosporaceae.}

Pittosporum crassifolium, Soland ex Putterd.

,

99

Tobira, Dryand.

, var. fol, varieg. Hort.

\section{Plantaginaceae.}

$\mathrm{N} \dagger$ Plantago albicans, Linn.

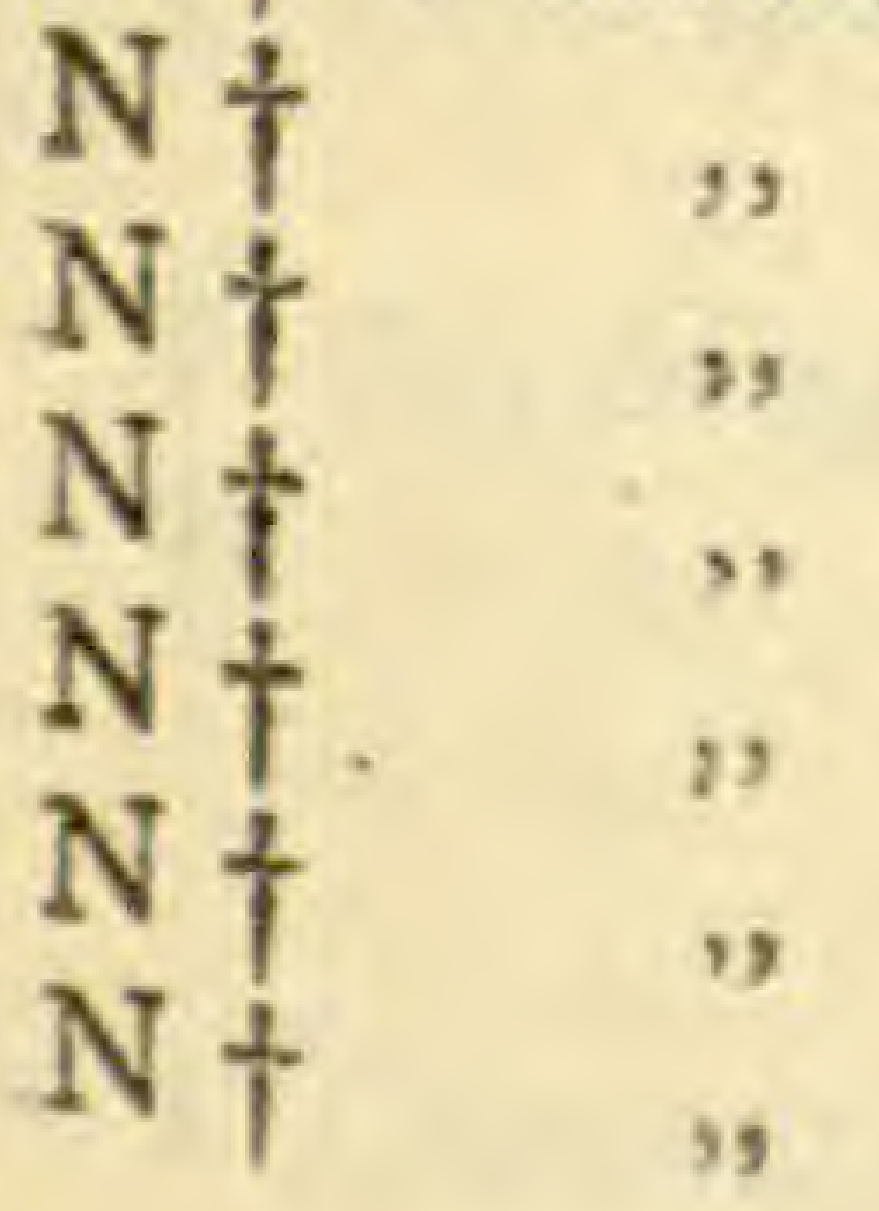

Coronopus, Linn.

Lagropus, Linn.

lanceolata, Linn.

major, Linn.

Psyllium, Linn.

serraria, Linn.

\section{Plumbaginaceae.}

Ceratostigma plumbaginoides, Bunge.

$\dagger$ Plumbago capensis, I hunb.

† $" \quad$ zeylanica, Linn.

$\mathrm{N} \dagger$ Statice reticulata, Linn.

$\dagger \quad, \quad$ sinensis, Girard.

$\dagger \quad$ Suworowi, Regel.

\section{Polemoniaceae.}

Cobaea scandens, Cav.

Gilia tricolor, Benth.

† Phlox Drummondi, Hook.

† ". Stellaria, A. Gray.

Polemonium mexicanum, Cerv. ex Lag.

Poligalaceae.

N Polygala monspeliaea, Linn. 


\section{Polygonaceae.}

$\dagger$ Antigonon leptopus, Hook.

$\mathrm{N}+$ Emex spinosa, Linn.

var: leucasteri, Hort.

Fagopyrum esculentum, Moench.

, tataricum, Gaertn.

Muechlembechia adpressa, Meissm.

$\mathrm{N} \dagger$ Polygonum aviculare, Linn.

articulintum, Linn.

$\mathrm{N}+\quad$ " Baldschuanichum, Regel.

N "Convolvulus, Linn.

$\mathrm{N}+\quad$, maritimum, linn.

" laprithitolium, Linn.

N Rumex bucephrlophorus, Linn.

" conglomeratus, Murr.

" pulcher, Linn.

"spec.

\section{Portulacaceae.}

† Portulaca grandiffora, Hook. $\mathrm{N}+\quad " \quad$ oleracea, Linn.

Portulacaria afra, Jacq.

\section{Primulaceae.}

$\mathrm{N} \dagger$ Anagallis arvensis, Linn.

$\mathrm{N}+$

$\dagger$ Cyclamen persicum, Mill.

var. punicea, $\mathrm{D}, \mathrm{C}$.

Primula grandiflora, I.am.

" Kewensis, Hort.

". malacoides, Franch.

" obconica, Hance.

" sinensis, Sabine. ex Lindl.

$\mathrm{N}+$ Samolus Valerandi, Linn. 


\section{Proteaceae.}

Grevillea robusta, A. Cunn in R. Br.

\section{Ranunculaceae.}

Aconitum Napellus, Linn.

$\mathrm{N} \dagger$ Adonis microcarpus, D. C.

$\mathrm{N} \dagger$ Anemone coronaria, Linn.

$\mathrm{N}$ hortensis, Linn.

$\dagger$ Aquilegia chrysantha, A. Gray.

$\dagger$, Skinneri, Hook.

" superba, Hort.

$\mathrm{N}+$ Clematis cirrhosa, Linn

$$
\text { Vitalba, Linn. }
$$

Cimicifuga racemosa, Mult. 39 japonica, Spreng.

$\dagger$ Delphinium Ajacis, Linn.

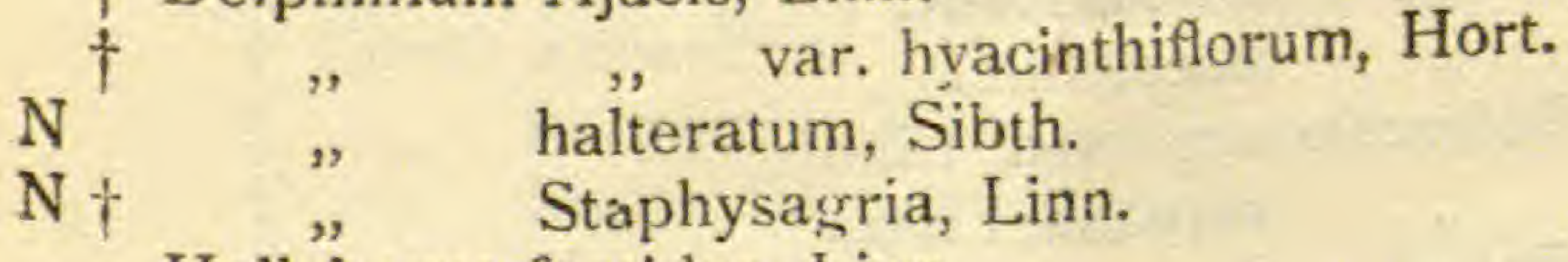

Helleborus foetidus, Linn. niger, Linn.

$\mathrm{N} \dagger$ Nigella damascena, Linn.

$\dagger$ " hispanica, Linn.

Ranunculus acris, Linn.

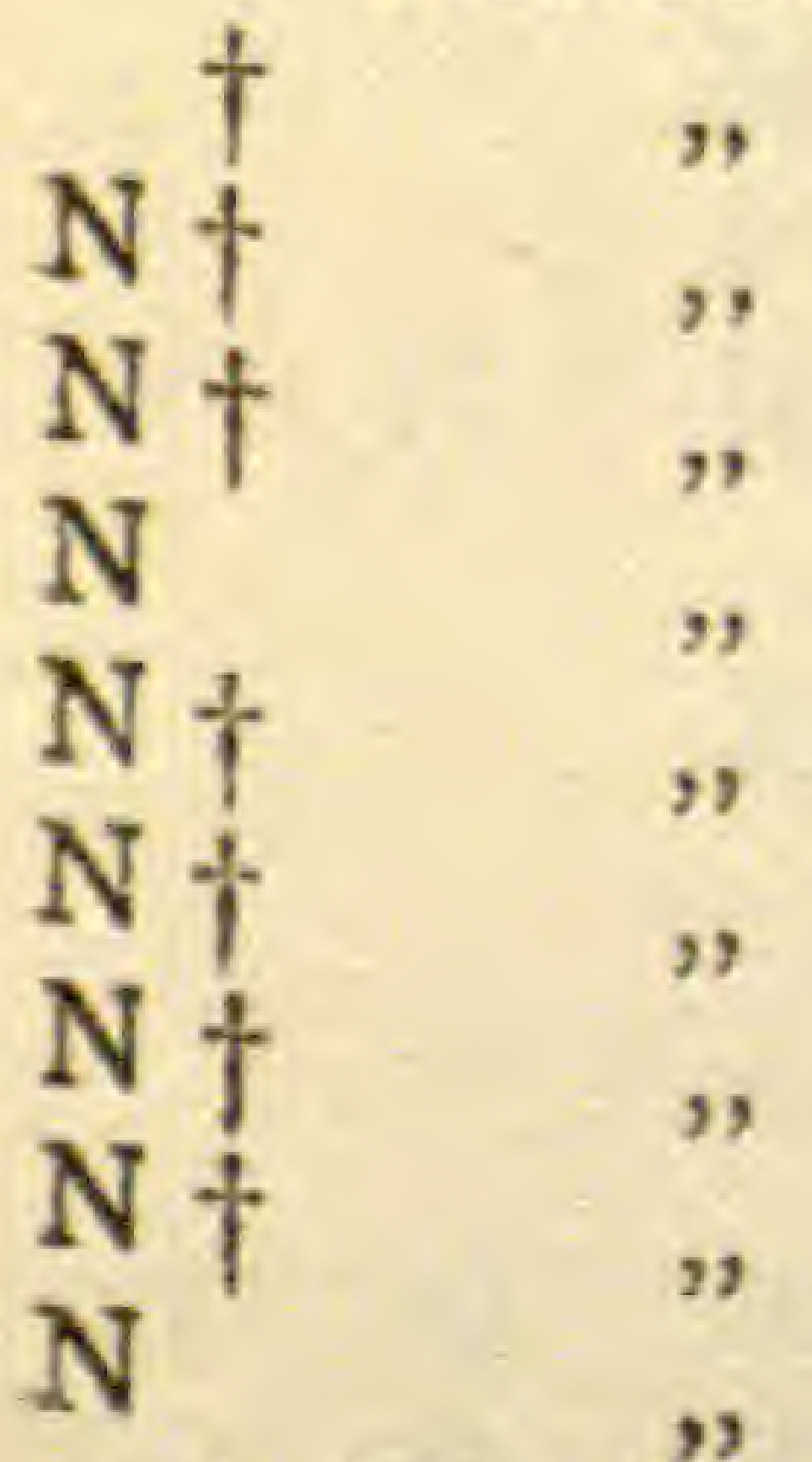

asiaticus, Linn.

arvensis, Linn.

bullatus, Linn.

diversifolius, Gilibert.

Ficaria, Linn.

flabellatus, Desf.

macrophyllus, Desf.

muricatus, Linn.

ophioglossifolus, Vill.

$\uparrow$ Thalictrum aquilegitolium, Linn. 
$\mathrm{N} †$ Reseda alba, Linn.

\section{Resodaceae.}

$\mathrm{N}$

$\begin{array}{ll} & \text { lutea, Linn. } \\ +\quad & \text { Luteola, Linn. } \\ & \text { odorata, Linn. }\end{array}$

Rhamnaceae,

† Colletia cruciata, Gill. et Hook.

N spinosa, Lam.

$\mathrm{N} \dagger$ Rhamnus Alaternus, Linn.

$\mathrm{N}$ oleoides, Linn.

$\mathrm{N} \dagger$ Zizyphus sativa, Gaertn.

Rosaceae.

$\mathrm{N}+$

Crataegus Azarolus, Linn.

toxyacantha Linn.

Eriobotrya japonica, Lindl.

Fragaria indica, Andr.

$\dagger$ Potentilla argentea, Linn,

$\mathrm{N}+$

reptans, Linn.

$\mathrm{N} \dagger$ Poterium Sanguisorba, Linn. Prunus Amygdalus, Stokes.

$\mathrm{N}$ Pyrus Sorbus, Gaertn.

- communis, Linn.

" Cydonia, Linn.

" Malus, Linn.

$\dagger$ Raphiolepis indica, Lindl.

Rosa Banksiae, R. Br, in Ait.

ovata, Briot.

$\begin{array}{ll}\mathrm{N} \dagger & \Rightarrow \\ \mathrm{N} \dagger \quad & \text { gallica, Linn. } \\ & \text { sempervirens, Linn. }\end{array}$

". Thea, Savi.

Spiraea cantoniensis, Lour. 


\section{Rubiaceae.}

$\mathrm{N} \dagger$ Asperula longiflora, W. \& Kit.

$\dagger$ Coprosmia lucida, Forst.

N Crucianella rupestris, Guss.

$\mathrm{N} \dagger$ Galium Aparine, Linn.

$\mathrm{N}$ saccharatum, All.

$\mathrm{N}+\quad, \quad$ tricorne, With.

Gardenia grandiflora, Lour.

Thunbergia, Linn. fil.

N Putoria calabrica, Linn. fil.

Rondeletia odorata, Jacq.

$\mathrm{N}+$ Rubia peregrina, Linn.

$\mathrm{N} \dagger$ Sherardia arvensis, Linn.

$\mathrm{N} \dagger$ Vaillantia muralis, Linn.

\section{Rutaceae.}

† Atalantia buxifolia, Oliv.

$\dagger$ Citrus Aurantiun, Linn.

$\dagger$, Bergamia, Risso.

$\dagger \quad$ " Bigaradia, Loisel.

$\dagger$ " Limonum, Risso.

$\dagger$, medica, Linn.

Corfea speciosa, Ait

Diosma ericoides, Linn.

Evodia hupensis, Forst.

Murraya exotica, Linn.

$\dagger$ Peganum Harmala, Lịnn.

$\mathrm{N} \dagger$ Ruta graveolens L. var. bracteosa, D. C. macrophylla, Soland, in Bat. Mag. Zanthoxylum alatum, Roxb.

\section{Salicaceae.}

N Poprulus alba, Linn.

$N$ Salix pedicellata, Desf. 
Sapindaceae.

$\uparrow$ Cardiospermum Halicacabum, Linn.

Dodonaea cuneata, Rudge.

† Koëlr Thunbergiana, Eckl. C. Zeyh. Melianteria paniculata, Laxm.

Melianthus major, L.inn.

† Sapindus Saponaria, Linn.

Sapotaceae.
† Pouteria suavis, Hort.

Saxifraga eae.
Deutzia scabra. Thunb, Hydrangea Hortensia, Siebold. Philadelphus coronarius, Linn.

$\mathrm{N}+$

$\mathrm{N}+$

$\mathrm{N}+$

$\mathrm{N}$

Alo siculum, Meria,

Scrophulariaceae.

Antirrhinum Asarina, Linn.

$\mathrm{N}$

Alonsoa grandiflora, Hort. majus, Linn.

Orontium,
diflora, Ho, Linn.

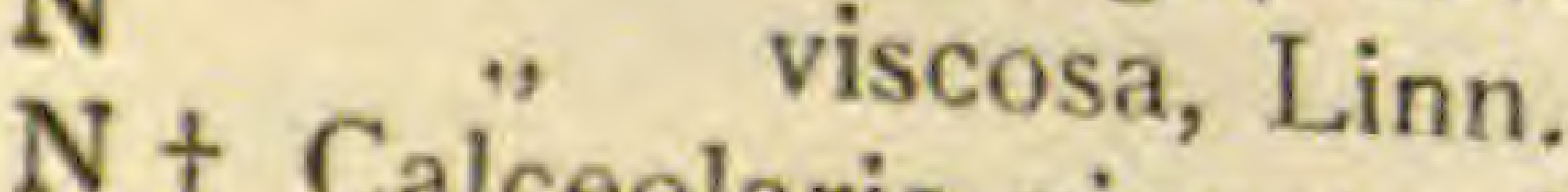

† Calceolaria pinnata, Linn.

$\mathrm{N}+$ Celsia cretica, Linn. Hort. Vilm.

" var. Arcturus, Hert.

Collinsia bicolor, Benth.

Digitalis purpurea, Linn.

$\mathrm{N}+$ Linaria commutata, Bernh,

$\mathrm{N}+\quad$, chalepensis, Linn

$\mathrm{N}+\quad$ " Cymbalaria, Linn. 
Linaria genistifolia, Mill. Gard. Dict.
$\mathrm{N}+\quad$ " multipunctata, Hof
$\mathrm{N} \dagger \quad$, pseudolaxiflora, Lojae.
$\mathrm{N} \dagger \quad, \quad$ spuria, Linn.
$\mathrm{N}+\quad, \quad$ triphylla, Linn.
Maurandia scandens, A. Gray.

Mimulus carinalis, Douglas. ex Benth.

luteus, Linn.

$\dagger \quad$.. tigrinus, Hort.

$\dagger$ Nemesia versicolor, E. Mey.

Penstemon barbatus, Roth. Catalect.

\section{Digitalis, Nutt.}

". gentianoides, Poir.

\section{Russellia juncea, Zucc.}

$\mathrm{N} \dagger$ Scrophularia aquatica, Linn.

$+$

$\begin{array}{ll}, & \text { orientalis, Lirn. } \\ , \quad & \text { peregrina, Linn. } \\ \text { sambucifulia, Linn. }\end{array}$

$\begin{array}{ccc} & \\ \mathrm{N} & & \text { Verbascum phoeniceum, Lin } \\ \mathrm{N} & \text { ", } & \text { Thuatum, Linn. }\end{array}$

$\mathrm{N} \dagger$ Veronica Andersonii, Lindl. \& Paxt.

$\mathrm{N}+\quad$ " Anagallis, Linn.

$\mathrm{N}+\quad " \quad$ arvensis, Linn.

† $\quad$ Proelta, Willd.
$\quad$ salicifolia, Forst. fil.

\section{Selaginaceae.}

Globularia vulgaris, Linn.

\section{Simarubiaceae.}

$\mathrm{N} \dagger$ Ailanthus glandulosa, Desf. Cneorum tricoccom, Linn. 


\section{Solanaceae.}

$\dagger$ Atropa Belladonna, Linn.

$\uparrow$ Browallia demissa, Linn.

Brunfelsia macrophylla, Benth. in D. C.

† Capsicum annuum, Linn.

$\dagger \quad, \quad$ longum, D. C.

microcarpum, Link.

minimum, Roxt.

pendulum, Willd.

var. fr. luteo, Hort.

Cestrum aurantiacum, Lindl.

" elegans, Schlecht.

" fastigiatum, Jacq.

" Parqui, L'Her.

Datura arborea, Linn.

" cornigera, Hook.

$\dagger \quad$ " chlorantha, Hook.

" ferox, Linn.

$\dagger$ fastuosa, Linn.

Metel, Linn.

$\mathbf{N}+\quad$ " $\quad$ quercitolia, H. B. et K.

$\mathrm{N}+$ Hyoscyamus albus, Linn.

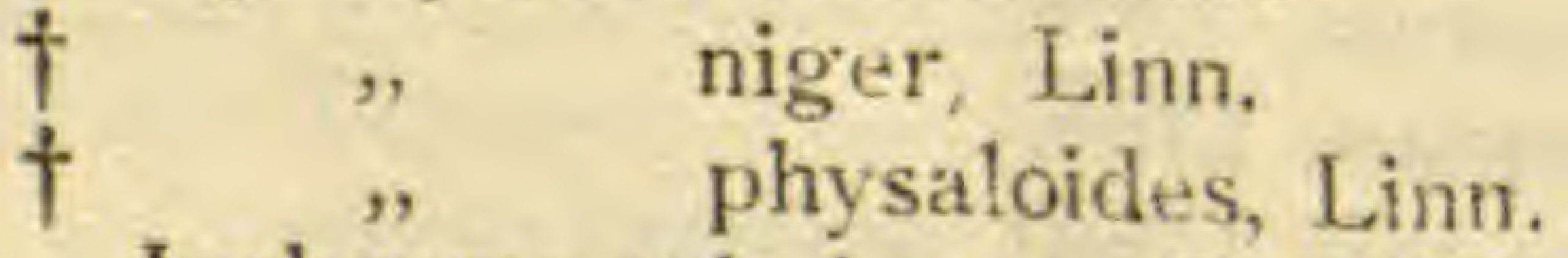

Iochroma tubulosum, Benth.

Juanulloa aurantiaca, Otto \& Dietr.

$\mathrm{N}+$ Lycium chinense, Mill.

$\mathrm{N}$

europeum, Linn.

† Lycopersicum esculentum, Hill.

$\uparrow$ Nicotiana alata, Link \& Otto.

$\mathrm{N} \dagger$

glauca, R. Graham.

rustica, Linn.

Tabacum, Linn.

virginica, Agardth.

$\mathrm{N} \uparrow$ Nycandra physaloides, Gaertn. 
$\dagger$ Petunia hybrida, Hort. Vilm.

$\dagger$ " nyctaginiflora. Juss.

$\mathrm{N} \dagger$ Physalis peruviana, Linn.

$+\quad$ var. pubescens, Linn.

† Salpiglossis sinuata, Ruitz. \& Pavon.

$\uparrow$ Schizanthus pirnatus, Ruitz. \& Pavon.

Solandra grandiflora, Sweet.

Solanum arbc reum, Humb. et Bonpl.

Dombeyi, Dun. in D. C.

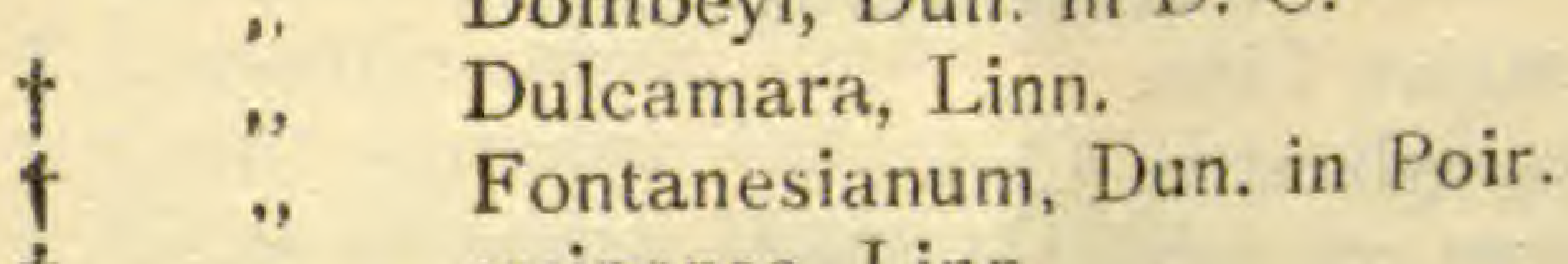

guinense, Linn.

Melongena, Linn.

var. ovigerum, Dun.

$\mathrm{N}+\quad " \quad$ nigrum, Linn.

$\mathrm{N}+\quad$ " $\quad \begin{aligned} & \text { robustum, H. Wendl. } \\ & \text { sodomeum, Linn. }\end{aligned}$

pseudo-capsicum, Linn.

, tuberosum, Linn.

" verbascifolium, Linn.

" Warszewiczii, Hort. ex Lambertye.

Wendlandii, Hook.

$\dagger$ Withania somnifera, Dun.

\section{Sterculiaceae.}

$\dagger$ Cola heterophylla, Schott.

† Sterculia acerifolia, A. Cunn.

$\dagger$ " platinifolia, Linn.

\section{Tamaricaceae.}

N Tamarix africana, Poir.

N " gallica, Linn. 


\section{Ternstroemiaceae.}

Camellia japonica, Linn.

\section{Tiliaceae.}

$\dagger$ Corchorus olitorius, Linn.

Sparmannia africana, Linn.

\section{Umbelliferae.}

$\mathrm{N} \dagger$ Ammi majus, Linn.

Angelica sylvestris, Linn.

$\dagger$ Anthriscus cerefolius, Hoffm.

$\mathrm{N}+$ Apium graveolens, Linn.

$\mathrm{N}+$ Bitora testiculata, Linn.

$\mathrm{N} \dagger$ Bupleurum subovatum, Link.

Carum Carvi, Linn.

$\mathrm{N} \dagger$, Petroselinum, Benth. \& Hook.

† Conium maculatum, Linn.

Coriandrum sativum, Linn.

$\mathrm{N}+$ Crithmum maritimum, Linn.

$\dagger$ Cuminum Cyminum, Linn.

$\mathrm{N}+$ Daucus Carota, Linn.

$\mathrm{N}$

Gingidium, Linn.

$\mathrm{N}$ Eryngium maritimum, Linn.

$\mathrm{N} \dagger$ Foeniculum piperitum, Sweet.

$\mathrm{N}+$

vulgare, Mill.

$\mathrm{N} \dagger$ Ferula communis, Linn.

$\mathrm{N}$

var: glauca, Linn.

$\mathrm{N}+$ Heliosciadum nodiflorum, Linn.

N Kundmannia sicula, Linn.

Levisticum officinale, Koch. in Nov.

$\mathrm{N}$ Oenanthe globulosa, Linn.

$\mathrm{N} \dagger$ Orlaya maritima, Gouan.

† Pimpinella Anisum. Linn.

N Ptychotis ammoides, Linn. 
N Ridolfia segetum, Linn.

$\mathrm{N}+$ Scandix Pecten-veneris, Linn.

$\mathrm{N} \dagger$ Smyrnium Olusatrum, Linn.

$\mathrm{N}+$ Tordylium apulum, Linn.

$\mathrm{N}+$ Torilis nodosa, Linn.

\section{Urticaceae.}

Boehmeria argentea, Linden, in Belg.

† Cannabis sativa, Linn.

Celtis rustralis, Linr.

Ficus altissima, Blume, Bijdr.

"Beniamina, Linn.

" Carica, Linn.

" citrifolia, Hort. ex Lam.

", dealbata, Hort. ex Hèrincq.

„, elastica, Roxb. Hort.

" " var, fol, varieg. Hort.

"Hookeri, Sweet, Hort. Brit.

" lucida, Dryand. in Ait. Hort, Kew.

" macrophylla, Desf.

", magnoliaefolia, Blume.

" repens, Rottl.

" senegalensis, Miq. 1. c.

,Vogeiii, Miq. I. c.

" spec.

Humulus japonicus, Sieh, \& Zucc.

Lupulus, Linn.

"Maclura aurantiaca, Nutt.

Morus alba, Linn.

" $\quad$ pendula, Hort.

" cucullata, Banaf. Sul. Gels.

nigra, Linn.

$\mathrm{N} \dagger$ Parietaria officinalis, Linn.

Pilea muscosa, Lindl.

$\mathrm{N} \dagger$ Theligonum Cynocrambe, Linn. 
N Ulmus campestris, Linn.

$\mathrm{N}+$ Urtica pilulifera Linn.

$\mathrm{N} \dagger \quad "$ urens, Linn.

\section{Valerianaceae.}

$\mathrm{N} \dagger$ Centranthus Calcitrapa, Linn

$\dagger \quad$ macrosiphon, Boiss.

$\dagger \quad$ " var, fl, albo, Hort.

$\dagger \quad, \quad$ ruber, D. C.

$\mathrm{N}+$ Fedia Cornucopiae, Gaertn.

Valeriana officinalis, Linn.

$\dagger$ Valerianelli carinata, Loisel.

$\mathrm{N}+$

$$
\text { eriocarpa, Desv. }
$$

\section{Verbenaceac.}

Callicarpa americana, Linn.

Cytharaexylon quadrangulare, Jacq.

Clerode alron fallax, Lindl.

fragrans, Willd.

$\dagger$ Duranta Plumierii, Jucq.

$\dagger$ Lantana Camara, Linn.

$$
\text { ". " var. fl. luteo, Hort. }
$$

Lippia citriodora, $\mathrm{H}$. B. et $\mathrm{K}$.

Verbena bonariensis, Linn.

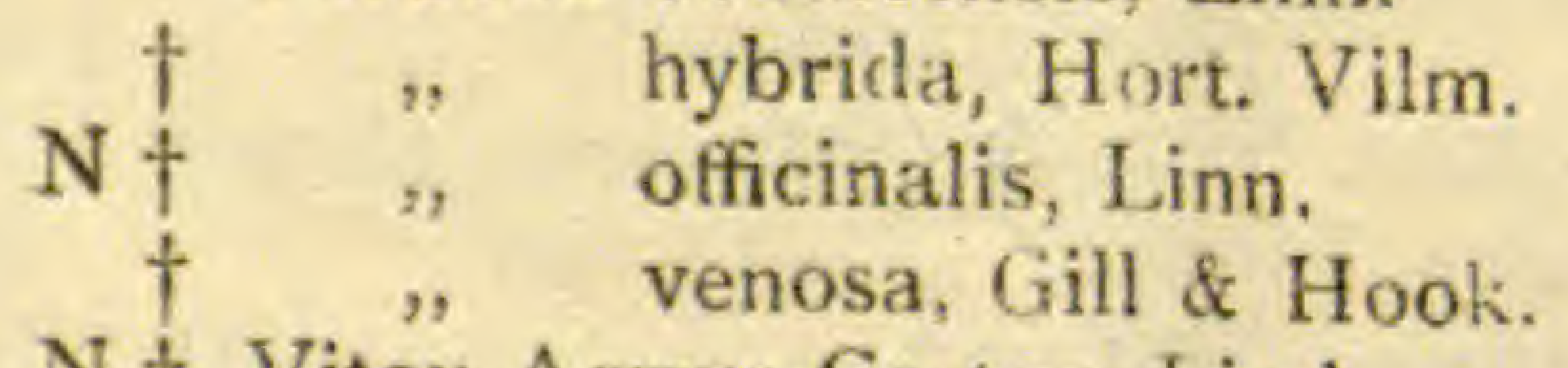

$\mathrm{N} \dagger$ Vitex Agnus-Castus, Lind.

\section{Violaceae.}

$\mathrm{N} \dagger$ Viola odorata, Linn.

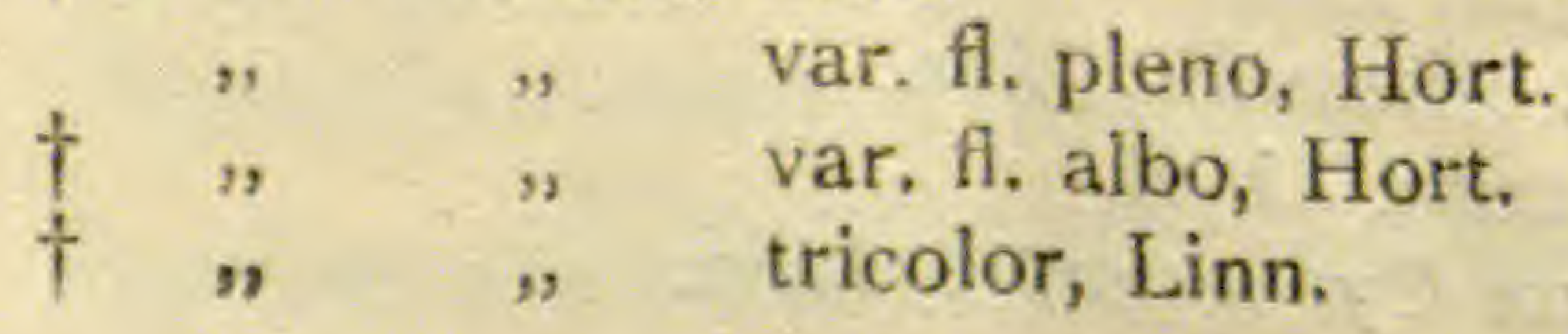




\section{Zygophyllaceae.}

N Fagonia cretica, Linn.

$\mathrm{N} \dagger$ Tribulus terrestris, Linn.

$\dagger$ Zygophyllum Fabago, Linn.

\section{MONOCOTYLEDONES.}

Alismaceae.

$\mathrm{N} \dagger$ Alysma Plantago, Linn.

N Damasonium Alisma, Mill.

\section{Amaryllidaceae.}

Agave americana, Linn.

" " var. fol. variegata, Hort.

" applanata, Lem. ex Jacobi.

" heteracantha, Zuce.

" mitraeformis, Jacobi.

, ingens, C. Kock.

" Lindleyi, Jacobi. in Abh.

" polyacantha, Haw.

" vivipara, Linn.

Alstroemeria pulchella, Lin. Fil.

$\dagger$ Amaryllis Belladonna, Linn.

Clivia nobilis, Lindl.

Crinum longifolium, Thumb.

Moorei, Hook.

Doryanthes Palmieri, W. Hill.

Eucharis grandiflora, Ph. \& Linden.

Furcraea Bedinghausii, C. Kock.

Háemanthus albiflos, Jacq. Hort.

coccineus, Linn.

" tigrinus, Jacq.

† Hippeastrum vittatum, Herb. Hort.

Leucojum vernum, Linn. 
Narcissus incomparabilis, Mill. Gard. Diet. Jonquilla, Linn. odorus, Linn. moschatus, Linn. poeticus, Linn.

$\begin{array}{ll}\mathrm{N}+\quad " \quad \text { var: fl. pleno, Hort. } \\ \mathrm{N} \quad \text { " } & \text { Tazetta, Lois. }\end{array}$

$\mathrm{N} \dagger$

Pancratium caribeum, Linn. maritimum, Linn.

Polianthes tuberosa, Linn.

Sternl,eryia lutea, Ker-Gawl.

$\dagger$ Vallota purpurea, Herb.

\section{Araceae.}

Acorus Calamus, Linn.

N Arisarum vulgare, Targ-Tozz.

N Arum italicum, Mill.

, palaestinum, Boiss.

Caladium esculentum, Vent

N Colocasia antiquorum, Schott.

N Dracunculus vulgaris, Schott.

Monstera deliciosa, Liebn.

Philodendron imbe, Schott. in Wien.

verricosum, Mathieu.

Pothos aureus, Linden.

\section{spec.}

+ Richardia africana, Kunth.

\section{Bromeliaceae.}

Aechmea bromeliaefolia, Baker. ex Benth. Billbergia thyrsoidea, Mart.

Karatas fulgens, Antoine.

Pitcairnia Altensteinii, Lem. in Fl. des Serres. Pourretia mexicana, Ruitz. \& Pavon. 


\section{Commelinaceae.}

† Tradescantia discolor, L'Her.

$$
\text { repens, Vend. in Roem. }
$$

Zebrina pendula, Schnizl.

\section{virginiana, Linn.}

\section{Cyperaceae.}

$\mathrm{N}+$ Carex distans, Linn.

$\mathrm{N} \dagger$, divisa, Huds.

N† "Schreberi, Schrank, Baier.

N $"$ serrulata, Biv.

$\mathrm{N}+$., vulpina, Linn.

Cyperus alternifolius, Linn.

$\mathrm{N} \dagger \quad, \quad$ esculentus, Linn. (bulbilli).

$\mathrm{N}+\quad$, longus, Linn.

†,$\quad$ Papyrus, Linn.

$\mathrm{N}+$ Galilea mucronata, Linn.

$\mathrm{N} \dagger$ Scirpus Holoschoenus, Linn.

$\mathrm{N}+\quad, \quad$ lacustris, Linn.

\section{Dioscoreaceae.}

Dioscorea divaricata, Blanco.

N Tamus communis, Linn.

\section{Equisetaceae.}

N Equisetum ramosissimum, Desf.

\section{Gnetaceae.}

Ephedra altissima, Desf.

N " distachya, Linn.

" fragilis, Desf.

\section{Graminaceae.}

$\mathrm{N} \dagger$ Aegilops ovata, Linn.

† Agropyrum campestre, Godr. \& Green. 
$\mathrm{N}+$ Agrostis verticillata, Vill.

$\mathrm{N}+$ Aira cupaniana, Guss.

$\dagger$," elegans, Forr. et Loisell.

N Ampelodesmos tenax, Link, Hort.

Andropogon distachyon, Linn.

$\dagger \quad$ " saccharatum, Moench.

$\mathrm{N} \dagger$ Anthoxanthum gracile, Bivon.

$\dagger \quad$ odoratum, Linn.

$\dagger$ Apera Spica-Venti, Beauv.

$\mathrm{N} \dagger$ Arundo Donax, Linn.

$\mathrm{N} \dagger \quad, \quad$ Pliniana, Tuira.

$\dagger$ Avena sativa, Linn.

$\mathrm{N} \dagger \quad$, sterilis, Linn.

Bambusa arundinacea, Willd.

" Fortunei, Hort. Van Houtte.

, nigra, Lodd. Cat.

Beckmannia erucaefolia, Host.

$\mathrm{N} \dagger$ Brachypodium distachyum, Linn.

$\dagger \quad$ " ramosus, Linn.

$\mathrm{N}+\quad, \quad$ sylvaticum, Huds.

$\mathrm{N} \dagger$ Briza maxima, Linn.

$\mathrm{N}+$, minima, Linn.

Bromus brizaefornis, Fisch \& May.

$\dagger \quad$ " ciliatus, Linn.

$\mathrm{N}+\quad$ macrestachyus, Desf.

$\mathrm{N}+$ madritensis, Linn.

$\mathrm{N} \dagger \quad$ " maximus, Desf.

" purgans, Linn.

$\dagger \quad$ " racemosus, linn.

† Chlorodopsis blanchardiana, Gay.

† Chrysopogon Gryllus, Trin.

† Coix Lachryma, 1 inn.

N Crypsis aculeata, Linn.

$\mathrm{N} †$ Cynodon Dactylon, Linn.

$\mathrm{N} \dagger$ Dactylis glomerata, Linn.

Eleusine indica, Gaertn. 
Eragrostis nebulısa, Hort.

$\mathrm{N} \dagger$ Festuca arundinacea, Schreb.

N Gastridium lendigerum, Vill.

$\dagger$ Gynerium argenteum, Nees.

$\uparrow$ Holcus lanatus, Linn.

$\uparrow$ Hordeum distachyon, Linn.

$\mathrm{N}+$ murinum, Linn.

$\mathrm{N} \dagger$ Koeleria phleoides, Vill.

$\mathrm{N}+$ Lagurus ovatus, Linn.

$\mathrm{N} \dagger$ Lamarchia aurea, Linn.

$\mathrm{N} \dagger$ Lepturus incurvatus, Linn.

$\mathrm{N}+$ Lolium perenne, Linn.

$\mathrm{N} \dagger \quad, \quad$ temulentum, Linn.

$\mathrm{N} \dagger$ Lygeum Spartum, Linn.

$\mathrm{N} \dagger$ Melica minuta, Linn.

$\mathrm{N} \dagger$ Milium multiflnrum, Cav.

Mischanthus sinensis, Andress.

$\mathrm{N} \dagger$ Panicum colonum. Linn. var. zebrina, Hort.

$\mathrm{N}+$

plicatum, Lam.

$\mathrm{N}+$ repens, Linn.

$\mathrm{N}+$ Pennisetum longistylum, Hochst.

† Phalaris canariensis, Linn.

$\mathrm{N}+\quad$ " coerulescens, Desí.

$\mathrm{N} \dagger \quad, \quad$ minor, Retz.

N ", nodosa, Murr.

$\mathrm{N} \dagger \quad$ " paradoxa, Linn.

N ", tuberosa, Linn.

$\mathrm{N}+$ Phragmites communis, Trin.

$\mathrm{N} \dagger$ Poa annua, Linn.

N ," bulbosa, Linn.

$\mathrm{N} \dagger$ Polypogon monspeliensis, Linn.

N Psamma arenaria, Linn.

Saccharum officinarum, Linn.

$\mathrm{N}+$ Scleropoa maritima, Linn.

$\mathrm{N}+\quad, \quad$ rigida, Linn. 
$\mathrm{N} \dagger$ Serrafalcus mollis, Linn.

$\mathrm{N} \dagger$ Setaria verticillata, Linn.

† Sorghum vulgare, Pers.

Spartina Schreberi, J. F. Gmel.

$\mathrm{N} \dagger$ Sphenopus divaricatus, Gouan.

N Sporobolus pungens, Schreb.

$\mathrm{N}+$ Stipa tortilis, Desf.

$\mathrm{N}+$ Trisetum aureum, Ten.

$\dagger$ Triticum sativum, Linn.

† Uniola latifolia, Michx. Fl. Bor.

$\mathrm{N} \dagger$ Vulpia ciliata, Pers.

$\dagger$ Zea Mays, Linn.

\section{Haemodoraceae.}

Sansevieria cylindrica, Boj. Hort. Maurit.

" thyrsiflora, Thunb.
$" \quad$ zeylanica, Willd.
" $\quad$ fol. varieg. Hort.

\section{Hydrocharidaceae.}

Vallisneria spiralis, Linn.

\section{Iridaceae.}

Anomatheca cruenta, Lindl.

$\mathrm{N} \dagger$ Antholyza aethiopica, Linn.

$\mathrm{N}+$ Crocus longiflorus, Raf.

" sativus, Linn.

Dierama cherifolia, C. Koch.

$\dagger$ Freesia refracta, Klatt.

† Gladiolus "gandavensis, X Van Houtte.
, var. Lemoine, Hort.

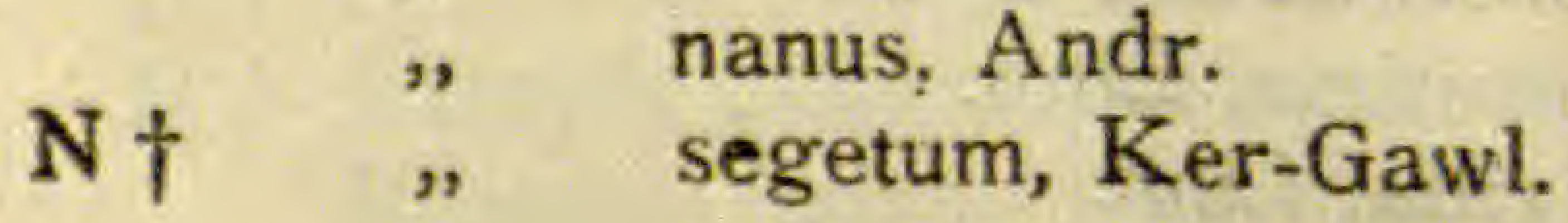


Iris aphylla, Linn.

" florentina, Linn.

", foetidissima, Linn.

$\mathrm{N} \dagger$, " germanica, Linn.

", hybrida. Retz.

" laevigata, Fisch. ex Fisch. \& Mey.

" pallida, Lam.

$\mathrm{N} \dagger \quad$ "Sisyrinchium, Linn.

" susiana, Linn.

", variegata, Linn.

" " var. aurea, Hort.

Xiphium, Linn.

Ixia paniculata, D. Dietr.

Libertia grandiflora, Sweet, Hort. Brit.

$\mathrm{N} \dagger$ Romulea Columnae, Seb. et Maur.

$\mathrm{N} \dagger \quad, \quad$ var. melitensis, Big.

, var. ramiflora, Ten.

Tigridia Pavonia, Ker-Gawl.

Tritonia crocata, Ker-Gawl.

, fenestrata, Ker-Gawl.

\section{Juncaceae.}

N Juncus acutus, Lian.

N " bufonius, Linn.

$\mathrm{N} \dagger \quad$, lamprocarpus, Ehrh.

\section{Liliaceae.}

† Agapanthus umbellatus, L'Her.

$\mathrm{N} \dagger$ Allium Ampeloprasum, Linn.

$\mathrm{N}$

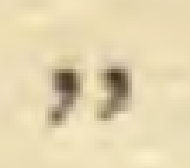

$\mathrm{N}$

$\mathrm{N}+$

$\mathrm{N}$
Chamaemoly, Linn.

neapolitanum, Cyr.

roseum, Linn.

sativum, Linn.

subhirsutum, Linn. 
Aloe arborescens, Mill.

" ciliaris, Haw.

$\mathrm{N}$

", Cooperi, Baker.

$\mathrm{N} \dagger$ Asphodelus fistulosus, Linn.

$\mathrm{N}+$ ramosus, Linn.

Asphodeline lutea, Reichb.

Aspidistra elatior, Blume.

N Asparagus aphyllus, Linn.

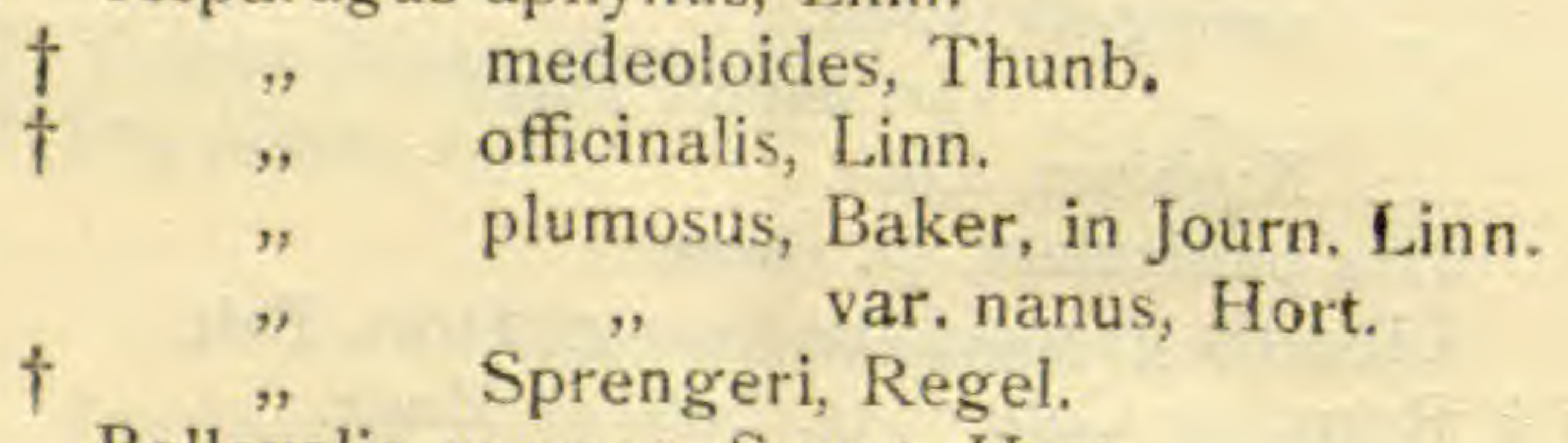

Bellevalia romana, Sweet, Hort.

N Caruelia arabica, Linn.

Chlorophytum Sternbergianum, Steu

N Colchicum montanum, Linn.

Convallaria majalis, Linn.

Dasylirion longissimum, Lem. ?

$\dagger$ Dianella coerulea. Sims.

seratifolium, Zucc. in Otto.

i " revoluta, $\mathrm{R}$. Br.

Dracaena Draco, Linn.

" Fontanesiana, Schult.

Gasteria angulata, Duval.

" disticha, Haw.

" spiralis, Baker.

"trigona, Haw.

Hawortia coarctita, Haw.

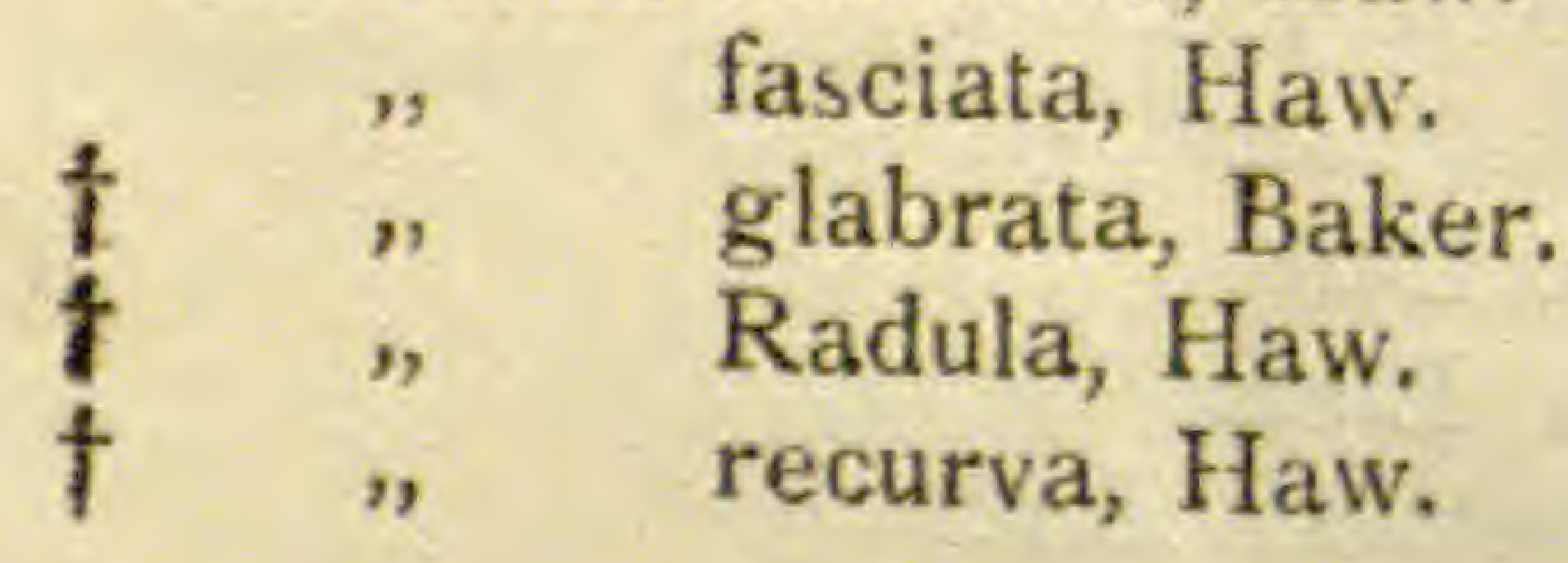


$\uparrow$ Hawortia spiralis, Duval. tesellata, Haw.

$\dagger$ Hemerocallis flava, Linn.

$+$

$$
\begin{aligned}
& \text { fulva, Linn. } \\
& \text { „, var. fl. pleno, Hort. }
\end{aligned}
$$$$
39
$$

Hyacinthus amethystinus, Linn.

orientalis, Linn.

23

var. fl. pleno, Hort.

Kniphofia aloides, Moench.

Lilium candidum, Linn.

"

lancifolium, Thunb.

" speciosum, Thunb.

tigrinum, Ker.Gawl.

Milla uniflora, R. Grah.

$\mathrm{N}+$ Muscari comosum, Linn.

$\mathrm{N}+$ " parviflorum, Desf.

$\mathrm{N}$

Nolina recurvata, Hensl.

$\mathrm{N} \dagger$ Nothoscordium fragrans, Vent.

$\mathrm{N}$

Ornithogalum narbonense, Linn.

Phormium tenax, Forst.

var. fol. varieg, Hort.

Rhodea japonica, Roth.

Ruscus aculeatus, Linn.

hypophyllum, Linn.

N Scilla autunnalis, Linn.

$\dagger$ " peruviana, Linn.

t sicula, Tineo.

N Smilax aspera, Linn.

N

var, angustifolia, Duthie.

". Sarsaparilla, Linn.
Tulipa gesneriana, Linn.
sylvestris, Linn.

N

". Sarsaparilla, Linn.
† Tulipa gesneriana, Linn.
sylvestris, Linn.

† Urginea maritima, Linn.

Veltheimia viridiflora, Jacq.

Veratrum nigrum, Linn. 
Yucca aloifolia, Linn.

" Atkinsi, Hort. ex Baker.
" $\quad$ Treculeana, Carr. in Rev. Hortie.

\section{Najadaceae,}

$\mathrm{N} \dagger$ Triglochin bulbosum, Linn.

Zannichellia palustris, Linn.

\section{Orchidaceae.}

Bletia hyacinthina, R. Br. in Ait,

Cypripedium Calceolus, Linn.

Dendrobium acuminatum, H. B. C. K.

N Ophrys apifera, Huds.

bombyliflora, Link.

" $\quad$ fusca. Link.

", lutea, Cav.

"speculum, Link,

Orchis coriophora, Linn.

" lactea, Poir.

" longicruris, Link.

" pyramidalis, Linn.

" var. Urvilleana, Fiori.-O. Urvilleana Somm, non Steud.

N " saccata, Ten.

N Serapias occultata, Gay.

$\mathrm{N}$ Spiranthes autumnalis, Pers.

\section{Palmaceae.}

Caryota urens, Linn.

Chamaedorea elegans, Mart.

$\mathrm{N} \dagger$ Chamaerops humilis, Linn.

Cocos australis, Mart. 
Howea Belmoreana, Becc. Forsteriana, Becc.

Livistonia australis, Mart. " chinensis, R. Br.

$\dagger$ Phoenix canariensis, Hort.

$\dagger$ " dactylifera, Linn.

$\dagger$ „, reclinata, Jacq.

" Roebelinii, Hort.

$\dagger$, rupicola, T. Anders.

$\uparrow$ Pritchardia filifera, Linden. Rhapis flabelliformis, L'Her.

$\uparrow$ Sabal Adansonii, Guerns. Trachycarpus excelsus, H. Wendl.

\section{Pandanaceae.}

Pandanus utilis, Bory.

\section{Pontederiaceae.}

Eichornia crassipes, Kunth. Pontederia cordata, Linn.

Scitaminaceae.

Alpinia nutans, Roscoe.

$\dagger$ Canna indica, Linn. lutea, Mill.

† Clinogyne grandis, Benth. et Hook. Hedychium Gardnerianum, Rose. Strelitzia Augusta, Thunb. " parviflora, Dryand, in Ait. " Reginae, Banks. in Ait.

$\dagger$ Thalia dealbata, Fras.

$\mathrm{N} \dagger$ Sparganium ramosum, Huds.

$\mathrm{N}$ Typha angustifolia, Linn. latifolia, Linn. 


\section{ACOTYLEDONES.}

\section{Filices.}

Adiantum Bauseii, Hort.

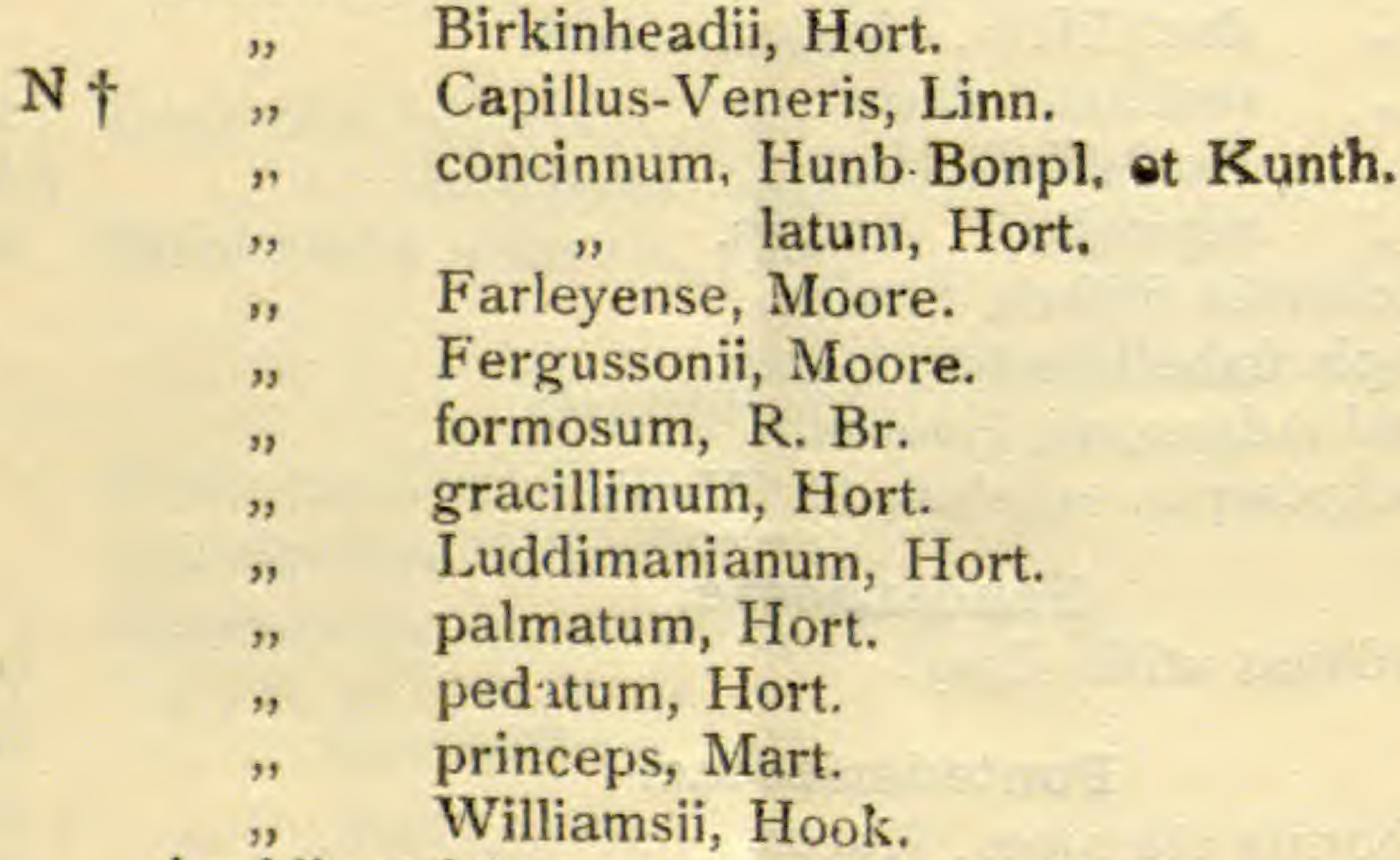

Aspidium falcatum caryotideum, Wall. Asplenium australasicum, Hook.

" marinum, Linn.

Nephrolepis cordifolia, Presl.

23

93

,

9, exaltata, Schott. pectinata, Sehott.

pluma, Moore.

Platycerium alcicorne, Desv.

$\uparrow$ Pteris longifolia, Linn.

Victoriae, Hort,

Selaginella Martensii, Hort.

C. Peniza,

Keeper Argotti Botanic Gardens, 


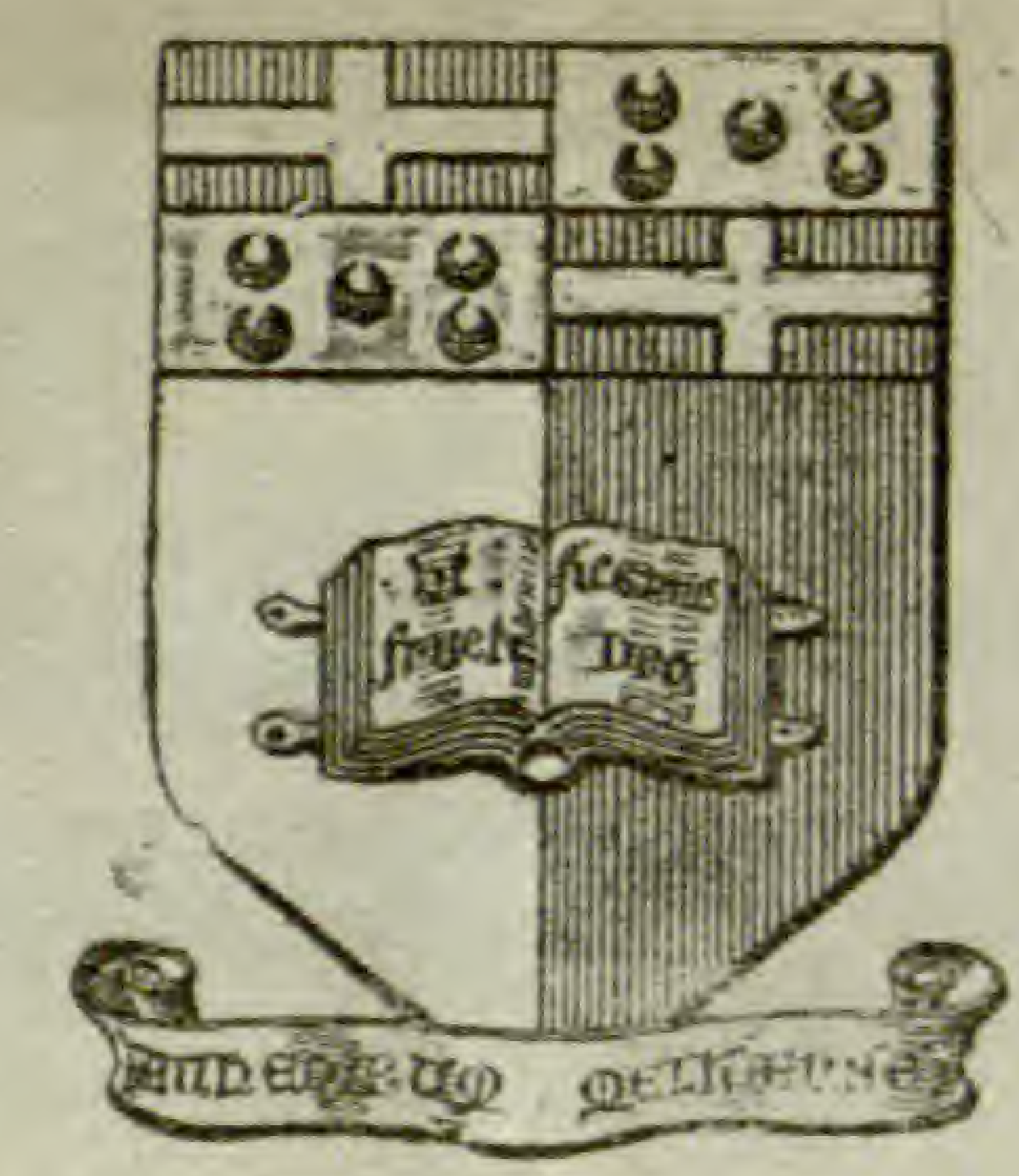

\section{THE BOTANIC GARDEN}

OF THE

ROYAL UNIVERSITY OF MALTA

\section{LIST OF SEEDS \\ FOR EXCHANGE}

COLLECTED IN THE YEAR 1937.

MALTA

GOVERNMENT PRINTING OFFICE 1938. 


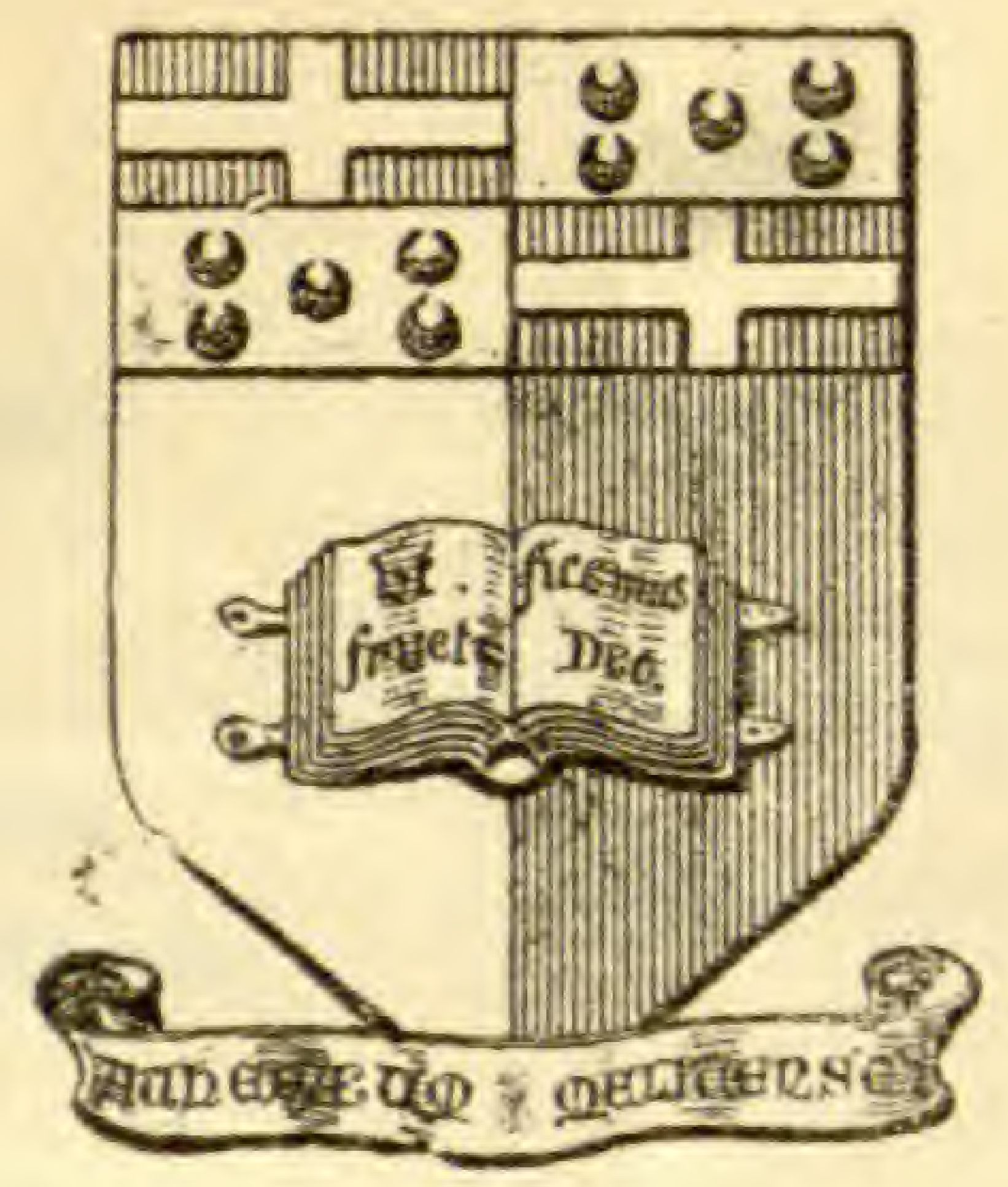

THE BOTANIC GARDEN

OF THE

ROYAL UNIVERSITY OF MALTA

\title{
LIST OF SEEDS
}

FOR EXCHANGE

COLLECTED IN THE YEAR 1937.

\author{
MALTA
}

GOVERNMENT PRINTING OFFICE

1938. 



\section{DICOTYLRDONEAE}

\section{Acanthaceae}

Acanthus mollis, L.

var, albiflorus, Borg.

Anisacanthus Wrightii, A. Gray.

Barleria cristata, L.

Justicia ventricosa, Wall.

\section{Amaranthaceae}

Amaranthus Caudatus, L.

, groecizans, L.

, salicifolius, Hort. Veitch.

, tricolor, $\mathbf{L}$.

Celosia cristata, L.

Gomphrena globosa, L.

\section{Ampelidaceae}

Vitis vinifera, L.

\section{Anacardiaceae}

Corynocarpus laevigata, Forst.

Pistacia Lentiseus, L.

Rhus toxicodendron, $\mathbf{L}$.

Schinus molle, L.

, terebinthifolis, Raddi.

\section{Apocynaceae}

Apocynum cannabinum, L.

Nerium Oleander, L.

Thevetia nereifolia, Juss, ex, Steud.

\section{Araliaceae}

Hedera Helix, L.

Sciadophyllum digitatum, G. Don.

\section{Aristolochiaceae.}

Aristolochia elegans, Mast.

$$
\text { , fimbriata, Cham. }
$$

\section{Asclepiadaceae}

Asclepias curassavica, L.

Cryptostegia madagaseariensis, Boj.

Gomphocarpus fruticosus, R. Br.

Vincetoxicum officinale, Moench

\section{Bignoniaceae}

Bignonia Tweediana Lind.

Tecoma Stans, Juss.

\section{Boraginaceae}

Anchusa italica, Retz.

Borago laxiflora, Willd.

,, officinalis, L.

Cerinthe aspera, Roth.

Echium parviflorum, Moench.

,$\quad$ plantagineum, $\mathbf{L}$.

Heliotropium europaeum, L.

Lithospermum apulum,, L.

Myosotis sylvatica, Hoffim.

Omphalodes linifolia, Moench.

Tournefortia Messerschimidia, Sweet.

\section{Cactaceae}

Opuntia Ficus-indica, Lab.

\section{Calycerae}

Acicarpha tribuloides, Juss.

\section{Campanulaceae}

Campanula Erinus, L.

Trachelium coerulum L.

\section{Capparidaceae}

Capparis spinosa, L. var rupestis, L.

Cleome graveolens, Rafin.

\section{Caprifoliaceae}

Lonicera implexa Soland.

\section{Cariophyllaceae}

Alsine procumbens, Valh.

Cerastium glomeratum, Thuill.

Dianthus caryophyllus, L. chinensis, L.

Gypsophyla elegans, Bieb.

Lychnis coeli-rosea, Desr. 
Saponaria officinalis, L.

Silene Behen, L. Githago, Scop.

,. fruticosa, L.

, nocturna, L.

, sedoides, Poir.

,. sericea All.

, sericea, All var albiflora Hort.

, vespertina, Retz.

Sagina apetala, Ard.

Spergularia rubra, L.

Stellaria media, L.

\section{Celastrineae}

Evonymus japonicus, L.

\section{Chenopodiaceae}

Atriplex hastatum, $\mathrm{L}$.

$$
\text { , Halimus, L. }
$$

Beta maritima, L.

Chenopodium amaranthicolor, Hort.

$$
\text { ,, murale, L. }
$$

Chenopodium olidum, Curt. opulifolium, Schrad.

Kochia arenaria, Roth, in Schrad. , tricophylla, Möllers.

Rhagodia nutans, $\mathrm{R}$. Br.

Salsola Soda, L.

Salsola Kali, L.

Suaeda maritima ,I .

\section{Compositae}

Achillea millefolium, L.

Ambrosia maritima, L.

Anthemis arvensis, L.

Artemisia Absinthium. L.

Astericus spinosus, L.

Bellis annua, L.

," peremis, $\mathbf{L}$.

,, sylvestris, Cyrill.

Calendula arvensis, L.

$$
\begin{gathered}
, \quad \text { officinalis, } \mathbf{L} \text {. } \\
, \text { var. fulgida, Raf. }
\end{gathered}
$$

Callistephus hortensis, Cass.

Carthamus lanatus, L.

$$
\text { , tinctorius, } \mathrm{L} \text {. }
$$

Catananche lutea, L.

Centaurea calcitrapa, L.

, Crassifolia, Bert.

," melitensis, L.

, nicaensis, All.
Carduus marmoratus, Boiss.

Chrysanthemum carinatum, Schoust.

segetum, L.
maximum, Ramond.
Parthenenium, Bernh.

Cineraria hybrida, Hrot.

Cichorium Intybus, L.

$$
\begin{aligned}
& \text {, } \quad \text { sumilum, Jacq. } \\
& \text { spinosum, L. }
\end{aligned}
$$

Conyza ambigua, D.C.

Coreopsis bicolor, Bosse.

Crepis rubra, L.

, parviflora, Desf.

Cynara Cardunculus, L.

,$\quad$ Scolymus; $\mathbf{L}$.

Gailardia pulchella, Fouger.

Galactites tomentosa, Moench.

Helianthus annuus, L.

, cueumerifolius, Turr. \&

Helychrysum rupestre, D.C.

Gray.

Helminthia echioides, L.

Hedypnois polymorpha, D.C.

Hedypnois polymorpha, D.C. var, tubaeformis, Tenore.

Hyoseris lucida, L.

$$
\begin{aligned}
& ,, \quad \text { radiata, I. } \\
& ,, \quad \text { seabra, L. }
\end{aligned}
$$

Tnula viscosa, L.

Lactuca saligna, L.

$$
, \quad \text { sativa, } \mathbf{L} \text {. }
$$

Leptosyne maritima, A. Gray, in

Melitella pusilla, Somm.

Matricaria chamomilla, L.

Mikania scandens, Willd.

Onopordon sipthorpianum Boiss.

Picridium vulgare Desf.

Phagnalon groceum, Boiss.

Rhagadiolus stellatus, L.

Senecio cineraria, D.C.

,, vulgaris, $\mathbf{L}$.

Scolymus hispanieus, L.

, maeulatus, $\mathrm{L}$.

Silybum marianum (L.) Gaertn.

Sonchus levis, Bartal.

, arper, Hill.

,. arper, Hill. 
Tagetes erecta, L. , patua, L.

Thrincia tuberosa, L.

Urospermum picriodes, 1. Xanthium spinosum, L.

Zimnia elegans, Jacq.

\section{Coniferae}

Callitris quadrivalis, Vent.

Pinus halepensis, Mill.

\section{Convolvulaceae}

Convolvulus arvensis, L. , pentapetaloides, $\mathrm{L}$. , tricolor, L.

Ipomaea rubro-coerulea, Hook.

\section{Crassulaceae}

Sedum rubens, L.

Umbilicus horizontalis, Guss.

\section{Cruciferae}

Biseutella didyma, L.

Alyssum maritimum, L.

Theris umbellata, L.

Lepidium Draba, L.

$$
\text { , Iberïs, } \mathbf{L} \text {. }
$$

Malcolmia maritima, R. Br.

Nasturtium officinale, R. Br.

Raphanus Landra, Moretti.

$$
\begin{aligned}
& , \quad \text { Raphanistrum, I. } \\
& , \quad \text { sativus, L. }
\end{aligned}
$$

Sisymbrium altissimum, L.

$$
\begin{array}{ll}
, & \text { Trio, I. } \\
, & \text { officinale, L. }
\end{array}
$$

Succovia balearica, Medik.

\section{Cucurbitaceae}

Bryonopsis laciniosa, Naud. Ecbalion Elaterium, A. Rich. Momordica charantia, L.

\section{Cupuliferae}

Quereus Ilex, L.

\section{Dipsaceae}

Scabiosa atropurpurea, $\mathbf{L}$. , maritima, $\mathbf{L}$.

\section{Ericaceae}

Erica multiflora, L.

\section{Euphorbiaceae}

Enphorbia aleppica, L.

$$
\begin{array}{ll}
" & \text { dendroides, L. } \\
" & \text { Ipecacuhana, L. } \\
\text { " } & \text { pinea, L. } \\
\text {, } & \text { Terracina, L. }
\end{array}
$$

Mercurialis annua, $\mathbf{L}$.

Ricinus communis, $\mathbf{L}$.

Ricinus communis, L.

$$
\text { v. singuineus, Hort. }
$$

\section{Ficoideae}

Mesembryanthemum cordyfolium, L. erystallinum, L. , nodiflorum, $\mathbf{L}$.

Tetragonia expansa, Murr.

\section{Frankeniaceae}

Frankenia intermedia, D.C.

\section{Fumariaceae}

Fumaria agraria, Lag. ,. officinalis, L.

\section{Geraniaceae}

Frodium malachoides, L.

Geranium dissectum, L.

$$
\text { ", } \quad \text { Rolle, L. }
$$

Pelargonium inquinans, Soland in Ait. Tropaeolum majus, L.

\section{Gentianaceae}

Erythraea pulchella, Sweet.

\section{Hydrophyilaceae}

Nemophilla insignis, Benth.

\section{Hypericaceae}

Hypericum tomentosum, L.

\section{Illecebraceae}

Herniaria cinerea, L.

Paronyehia argentea, L.

Polycarpon tetraphyllum, L. 


\section{Labiatae}

Ballota nigra, L.

Lamium amplexicaule, L.

Leonotis Leonurus, $\mathrm{R}$. Br.

Marrubium vulgare, $L$.

Mentha Pulegium, L.

Ocimum Basilicum, L. ,$\quad$ minimum, $\mathbf{L}$.

Prasium majus, L.

Satureja microphylla. D'Urville.

Salvia clandestina, L.

, officinalis, L.

Sideritis romana, L.

Stachys hirta, L.

Teucrium fruticans, L.

\section{Lauraceae}

Laurus nobilis, L.

\section{Leguminoseae}

Acacia cyanophylla, Lindl.

Anthyllis tetraphylla, L.

$$
\text { , vulneraria, L. }
$$

Astragalus boeticus, L. hamosus, I.

Caesalpinia Gillesii, Wall ex Hook.

Cassia emarginata, $\mathbf{L}$.

Ceratonia siliqua, $\mathbf{L}$.

Cercis siliquastrum, L.

Coronilla Emerus, L. scorpioides, L.

Ervum Ervilia, L. ,. gracile, Lois.

Gleditschia triacanthos, L.

Hedysarum coronarium, I.

Hymenocarpus circinatus, Savi.

Hippocrepis multisiliquosa, L.

Lathyrus annuus, $\mathbf{I}$. cicera, $\mathbf{L}$.

elymenum, $\mathbf{L}$.

Gorgoni Parl.

Ochrus, I.

odoratus, $\mathbf{I}$.

sphoericus, Retz.

Lotus edulis, L.

, = ornithopodioides, $\mathbf{L}$.
Medicago arborea, L.

,$\quad$ ciliaris, L.

, Echinus, D.C.

,. litoralis, Rohde.

,, lupulina, L.

,. orbicularis, L.

, scutellata, Mill.

, tuberculata, Willd.

Mimosa pudica, L.

Melilotus elegans, Salzam.

, indicus, All.

, italica, Lam.

, mossanensis, $\mathbf{L}$.

Ononis Sieberi, Besser.

" biflora, Desf.

, Natrix, L.

, reclinata, $\mathrm{L}$.

Pisum arvense, $\mathbf{L}$.

Psoralea bituminosa, L.

Scorpiurus subvillosa, I.

,, vermiculata, $\mathrm{L}$.

Spartium Junceum, L.

Tetragonolobus purpureus, L.

Trifolium agrarium, $\mathbf{L}$.

, cherleri, L.

, nigroescens, Viv.

,. resupinatum, $\mathrm{L}$.

, scabrum, L.

, stellatum, L.

,. tomentosum, L.

Trigonella Foenum-groecum, L.

Vicia "Faba, L. monspeliaea, $\mathbf{L}$.

, lutea, $\mathrm{L}$.

, narbonensis, L.

narbonensis, $\mathrm{T}_{\text {. }}$.

var, serratifolia, Jacq.

,, sativs, $\mathbf{L}$.

, , , var. cuneata, Guss.

\section{Linaceae}

Linum decumbens Desf.

, gallicum, $\mathbf{T}$.

,, grandiflorum, Desf.

, usitatissimum, $\mathbf{L}$.

\section{Lobeliaceae}

Lohelia Erinus, L. triquetra, $\mathbf{L}$. 


\section{Lythraceae}

Lythrum Hyssopifolia, L,

\section{Magnoliaceae}

Mignolia grandiflora, L.

\section{Malvaceae}

Abutilon avicennae, Gaertu.

Althaea rosea, Cav.

Gossypium herbaceum, I. religiosum, L.

Lagunaria Patersonii, L.

Lavatera arborea, L.

\section{Meliaceae}

Melia Azederach, L.

\section{Myoporineae}

Myoporum serratum, R. Br.

\section{Myrtaceae}

Myrtus communis, L.

Myrtus communis, $\mathbf{L}$. var. leiocarpa, Wright in Sauv.

\section{Nyctagineae}

Mirabilis Jalapa, L.

\section{Oleaceae}

Jasminum Azoricum, L.

Olea europea, L.

\section{Onagraceae}

$\mathrm{C}^{\mathrm{l}}$ erkia elegans, Dougl.

Epilobium Tournefortii, Michal.

Oenothera rosea, Soland in Ait.

\section{Oxalidaceae}

Oxalis cernua, Thumb. (bulbilli).

Oxalis, cernua, Thumb. var pleniflora, corniculata, L.

Ces. (bulbilli).

", hirta, L.

(bulbilli).

purpurea, L.

tenuifolia, Jacq.

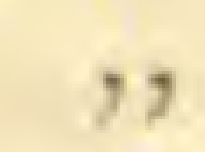

truncatula, Jacq.

, ,

violacea, $\mathbf{L}$.

\section{Papaveraceae}

Chelidonium majus, L.

Esescholtzia ealifornica, Cham.

Glaucium flavum, Crantz.

Hypecoum procumbens, L.

Papaver hybridum, I.

$$
\begin{aligned}
& \text { Rhoeas, L. } \\
& , \quad \text { setigerum, D.C. } \\
& \text {," somniferum, L. var. album, }
\end{aligned}
$$

Hort.

\section{Phytolaccaceae}

Phytolacca decandra, L.

\section{Pittosporaceae}

Pittosporum Tobira, Dryand.

\section{Plantaginaceae}

Plantago albicans, $\mathrm{L}$.

$\begin{array}{ll}\text { ", } & \text { coronopus, L. } \\ \text { Lagopus, L. } & \text { lanceolata, L. } \\ \text { ", } & \text { major, L. } \\ \text { major, L. var, rubra, Hort. } & \text { Psylium, L. } \\ \text { ", } & \text { serraria, L. }\end{array}$

\section{Plumbaginaceae}

Plumbago europea, L.

Statice reticulata, $\mathbf{L}$.

,. Suworowi, Regel.

\section{Polemoniaceae}

Phlox Drummondi, Hook

\section{Polygalaceae}

Polygala myrtifolia, L.

\section{Polygonaceae}

Antigonon leptopus, Hook.

Emex spinosa, L.

Polygonum aviculare, $\mathbf{L}$.

, convolvulus, $\mathbf{L}$.

Rumex bucephalophorus, $\mathbf{L}$.

, pulcher, L. 


\section{Portulaceae}

Portulaca grandiflora, Hook.

$$
\text { , oleracea, L. }
$$

\section{Primulaceae}

Anagallis arvensis, L.

Anagallis arvensis, I.

Samolus valerandii, L.

$$
\text { var. punicea, D.C. }
$$

\section{Ranunculaceae}

Adonis microcarpus, D.C.

Anemone coronaria, L.

$$
\text { , hortensis, L. }
$$

Clematis cirrhosa, L.

Delphinium ajacis, L.

$$
\text { , halteratum, Sibth. }
$$$$
\text { ,. Staphysagria, } \mathrm{L} \text {. }
$$

Isopyrum fumarioides, $\mathbf{L}$.

Nigella damascena, L.

$$
\text { , hispanica, } \mathbf{L} \text {. }
$$

Ranunculus arvensis, L.

$$
\begin{array}{ll}
, \quad & \text { bullatas, L. } \\
, \quad & \text { Ficaria, L. } \\
\text { flabellatus, Desf. }
\end{array}
$$

Ranunculus macrophyllus, Desf.

$$
\text { muricatus, } \mathrm{L} \text {. }
$$

Thalictrum aquilegifolium, L.

\section{Resedaceae}

Reseda alba, L.

$$
\begin{array}{ll}
\text { lutea, } \mathrm{L} . \\
, \quad & \text { luteola, L. } \\
& \text { odorata, L. }
\end{array}
$$

\section{Rhamnaceae}

Colletia spinosa, Lam.

Paliurus Spina-Christi, Mill.

Rhamnus Alaternus, L.

Zizyphus sativa, Gaertn.

\section{Rosaceae}

Crategus oxyacantha, L.

Poterium Sanguisorba, L.

Raphiolepis ovata, Briot.

Rosa gallica, L.

\section{Rubiaceae}

Coprosma lueida, Forst.

Galium aparine, L.

, saccharatum, All.

, tricorne, With.

Sherardia arvensis, L.

Vailantia muralis, L.

\section{Rutaceae}

Murraya exotica, L.

Ruta graveolens, $\mathbf{L}$.

\section{Sapindaceae}

Köerleuteria panículata, Laxm.

Melianthus major L. minor, $\mathrm{L}$.

Sapindus Saponaria, L.

\section{Sapotaceae}

Argania Sideroxylon, Roem. \& Shult.

\section{Scrophulariaceae}

Antirrhinum majus, I.

$$
\begin{aligned}
& , \quad \text { Orontium, L. } \\
& , \quad \text { siculum, Mreia. }
\end{aligned}
$$

Calceolaria pinnata, L.

Celsia cretica, L.

Linaria chalepensis, L.

,. cymbalaria, L.

, pseudolaxiflora, Lojac.

, triphylla, L.

Mimulus guttatus, Fise.

$$
\text { ,y tigrinus, Hort. }
$$

Serophularia aquatiea, L.

$$
\text { , } \quad \text { trifoliata, } \mathbf{L} \text {. }
$$

Verbascum phoeniceum, L.

Veronica Anagallis, L.

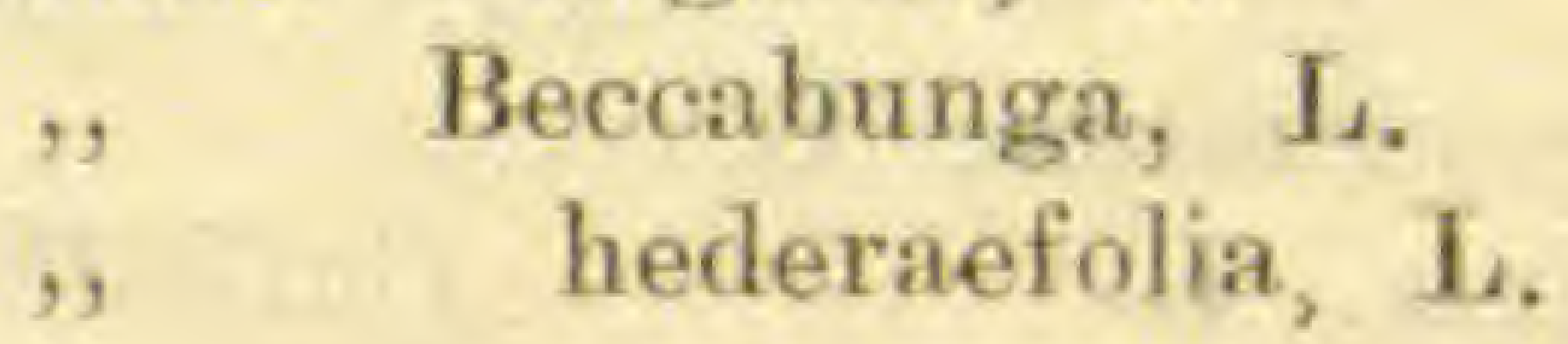

\section{Simarubiaceae}

Ailanthus glandulosa, Desf.

Cneorum tricoccon, $\mathbf{L}$. 


\section{Solanaceae}

Capsicum annum, L.

Datura chlorantha, Hook.

, fastuosa, $\mathbf{L}$.

,g ferox, L.

, Metel, L.

, quercifolia, H. B. et $\mathbf{K}$.

,, Stramonium, $\mathbf{L}$.

Hyosciamus albus, $\mathbf{L}$. niger, $\mathrm{L}$.

Lycium chinense, Mill.

Nicotiana alata, Link. \& Otto.

$$
\text { ,, glauca, R. Graham. }
$$

Nicandra physaloides, Gaertn.

Petunia hybrida, Hort.

Solanum Duleamara, L.

,, fontanesianum, Don. in Poir.

Solanum nigrum, L.

, ,, var. miniatum,

,, pseudocapsicum, L.

Bernh.

Withania somnifera, Don.

\section{Sterculaceae}

Cola heterophylla, Schott.

\section{Umbelliferae}

Apium graveolens, L.

Bifora testieulata, L.

Buplureum subovatum, Link.

Conium maculatum, $\mathbf{L}$.

Crithmum maritimum, L.

Daucus Carota, L.

Foeniculum vulgare, Mill.
Ferula communis, L.

Kundmannia sicula, L.

Oenanthe globulosa, L.

Scandix pecten-Veneris.

Tordylium apulum, $\mathbf{L}$.

Torylis nodosa, L.

\section{Urticaceae}

Cannabis sativa, L.

Parietaria officinalis, L.

Theligonum cynocrambe, L.

Urtica pilulifera, $\mathbf{L}$.

, urens, L.

\section{Valerianaceae}

Centranthus Calcitrapa, L. ruber, D.C.

Fedia cornucopiae, Gaertn.

Valerianella carinata, Loisel.

$$
\text { , eriocrapa, Desv. }
$$

\section{Verbenaceae}

Durantha Plumierii, Jacq.

Lantana camara, L.

Verbena hybrida, Hort. Vilm. officinalis, $\mathrm{L}$.

Vitex Agnus-Cactus, Lindl.

\section{Violaceae}

Viola tricolor, L.

\section{Zygophyllaceae}

Tribulus terrestris, L.

\section{MONOCOTYLEDONES}

\section{Alismaceae}

Alisma Plantago, L.

\section{Amaryllidaceae}

Narcissus serotinus, L. ,, Tazzetta, Loís. Pancratium maritimum, L.

\section{Araceae}

Arisarum vulgare, Targ-Tozz. Arum italicum, Mill. Richardia africana, Kunth.

\section{Commelinaceae}

Tradescantia virginiana, $\mathrm{L}$. 
Cyperaceae

Cyperus longus, $\mathrm{L}$.

\section{Dioscoraceae}

Dioscorea divaricata, Blanko.

\section{Gnetaceae}

Ephedera fragilis, Desf.

\section{Graminaceae}

Aegylops ovata, L.

Ampelodesma tenax, Link.

Anthoxanthum gracile, Bivon.

Avena sterilis, L.

Brachypodium distachyum, L. sylvaticum, Huds.

Briza maxima, L. ,, minima, L.

Bromus madritensis, L.

Bromus purgans, L.

Dactylis glomerata, L.

Eleusine indica, Gaertn.

Koeleria phleoides, Vill.

Lagurus ovatus, L.

Lamarckia aurea, L.

Lolium perenne, $\mathbf{L}$.

, temulentum, L.

Lygeum Spartium, L.

Milium multiflorum, Cav.

Pennisetum longystilum, Auct. non

Hochst.

Phalaris canariensis, L.

$$
\text { ,) minor, Retz. }
$$

Poa annua, $\mathbf{L}$.

Polypogon monspeliensis, L.

Psamma arenaria, $\mathbf{L}$.

Scleropoa rigida, L.

Serrafalcus mollis, $\mathrm{L}$.

Setaria verticillata, $\mathrm{L}$.

Sorghum vulgare, Pers.

Sphoenopus divaricatus, Gouan.

Trisetum aureum, Ten.

Triticum sativum, L.

Vulpia ciliata, Pers.

Zea Mais, L.

\section{Iridaceae}

Antholyza aethiopica, L.

Crocus longiftorus, Raf.

Fresia refracta, Klatt.

Gladiolus gandavensis, Van Houtte. , segetum, Ker-Gawl.

\section{Juncaceae}

Juncus Bufonius, L.

\section{Lemnaceae}

Lemna gibba, L.

,, minor, $\mathrm{L}$.

, trisulea, $\mathbf{L}$.

(pl. viv.)

,

,

\section{Liliaceae}

Allium roseum, L.

,, subhirsutum, L.

Asphodelus fistulosus, $\mathbf{L}$.

, ramosus, $\mathbf{L}$.

Asparagus medeoloides, Thunb.

,. Sprengeri, Regel.

Chlorophytum Sternbergianum, Steud.

Muscari comosum, L.

Nothoscordium fragrans, Vent.

Scilla peruviana, L.

Tulipa sylvestris, L.

Urginea maritima, I.

\section{Najadaceae}

Triglochin bulbosum, Guss.

\section{Orchidaceae}

Ophrys Fusea, Link.

Orchis coriophora, L.

\section{Palmeae}

Chamaerops humilis, L.

Phoenix eanariensis, Hort.

\section{Salvinaceae}

Azolla caroliniana,, Willd. (pl. viv.)

\section{Scitaminaceae}

Canna indica, $\mathbf{L}$.

Thalia dealbata, Fras.

\section{Typhaceae}

Sparganium ramosum, Huds.

Typha angustifolia, $\mathbf{L}$. 


\section{ACOTYLEDONES}

Filices

Adianthum Capillus-veneris, L.
Nephrolepis tuberosa Hook.

Pteris longifolia, $\mathbf{L}$.

Please address requests, possibly before the 31st of March, 1938, to:-

The Director,

Royal University Botanic Gardens,

Floriana, Malta.

S. L. VELLA,

Director of the Botanic Gardens and Professor of Botany.

C. Penza,

Keeper. 